Supplement of Clim. Past, 16, 29-50, 2020

https://doi.org/10.5194/cp-16-29-2020-supplement

(C) Author(s) 2020. This work is distributed under

the Creative Commons Attribution 4.0 License.

(c) (1)

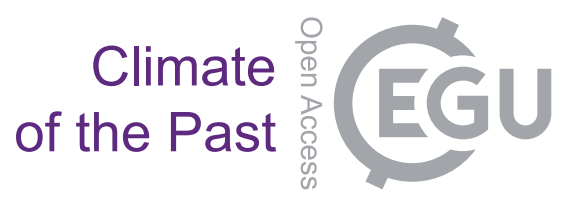

Supplement of

\title{
NALPS19: sub-orbital-scale climate variability recorded in northern Alpine speleothems during the last glacial period
}

Gina E. Moseley et al.

Correspondence to: Gina E. Moseley (gina.moseley@uibk.ac.at)

The copyright of individual parts of the supplement might differ from the CC BY 4.0 License. 


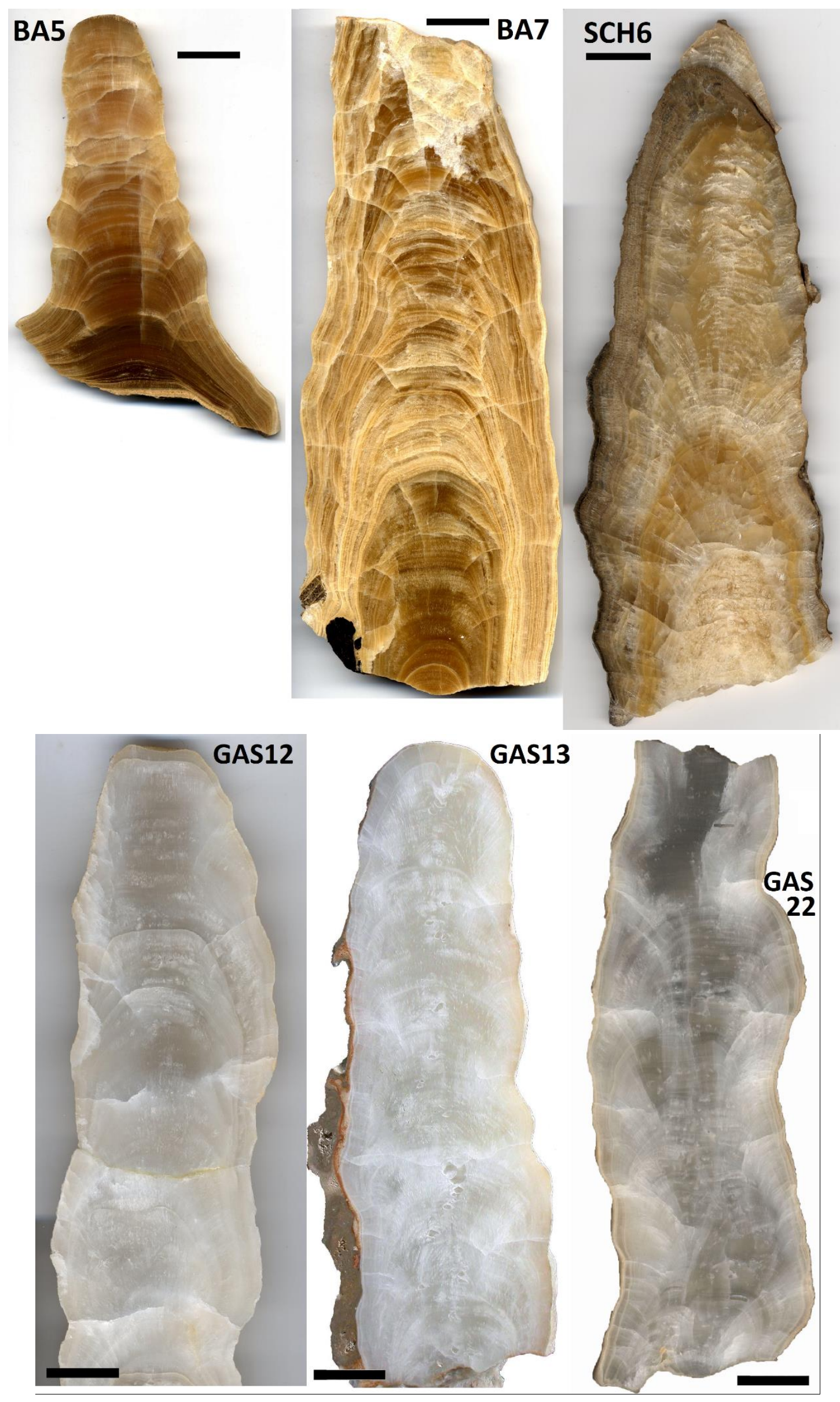




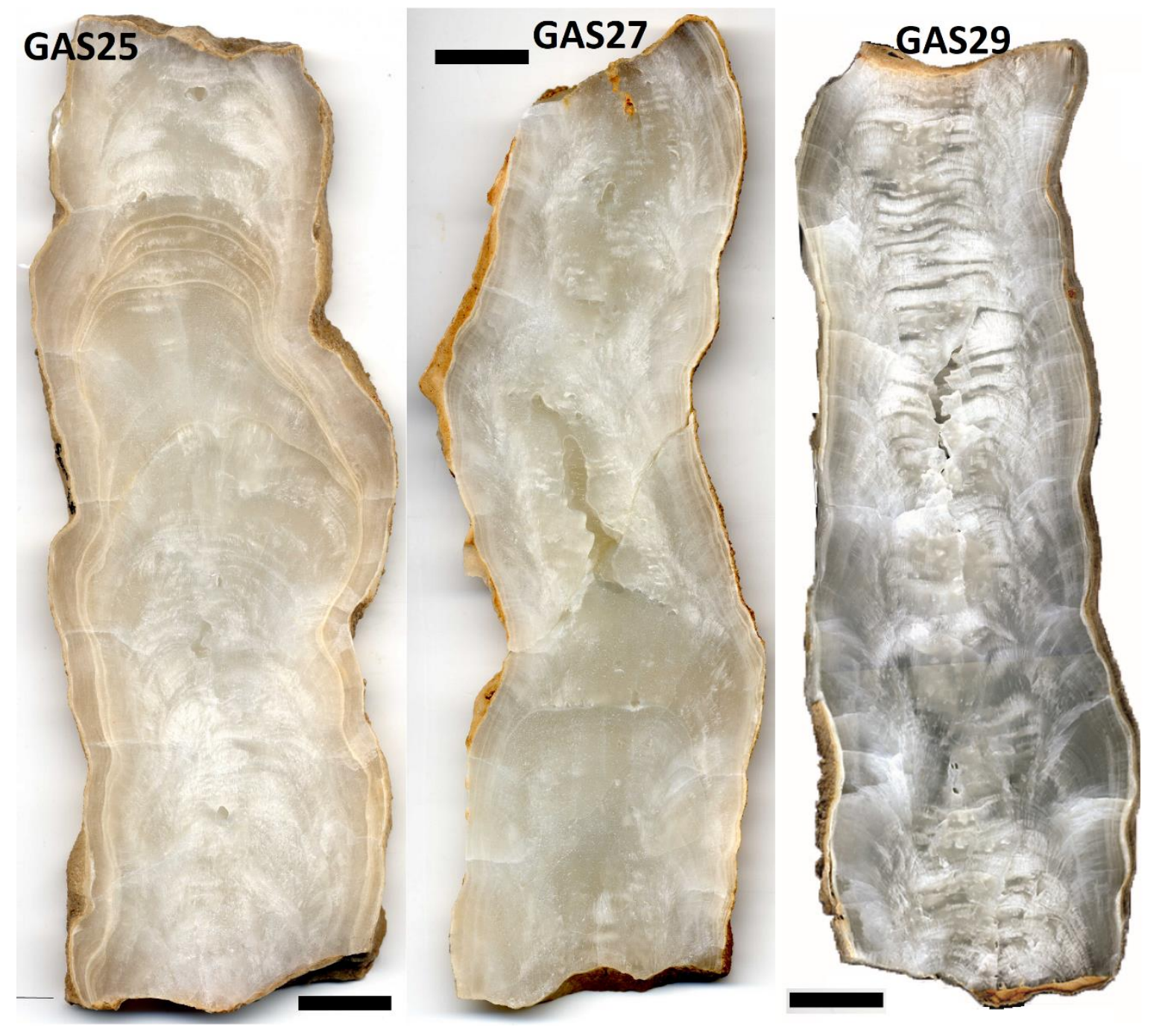




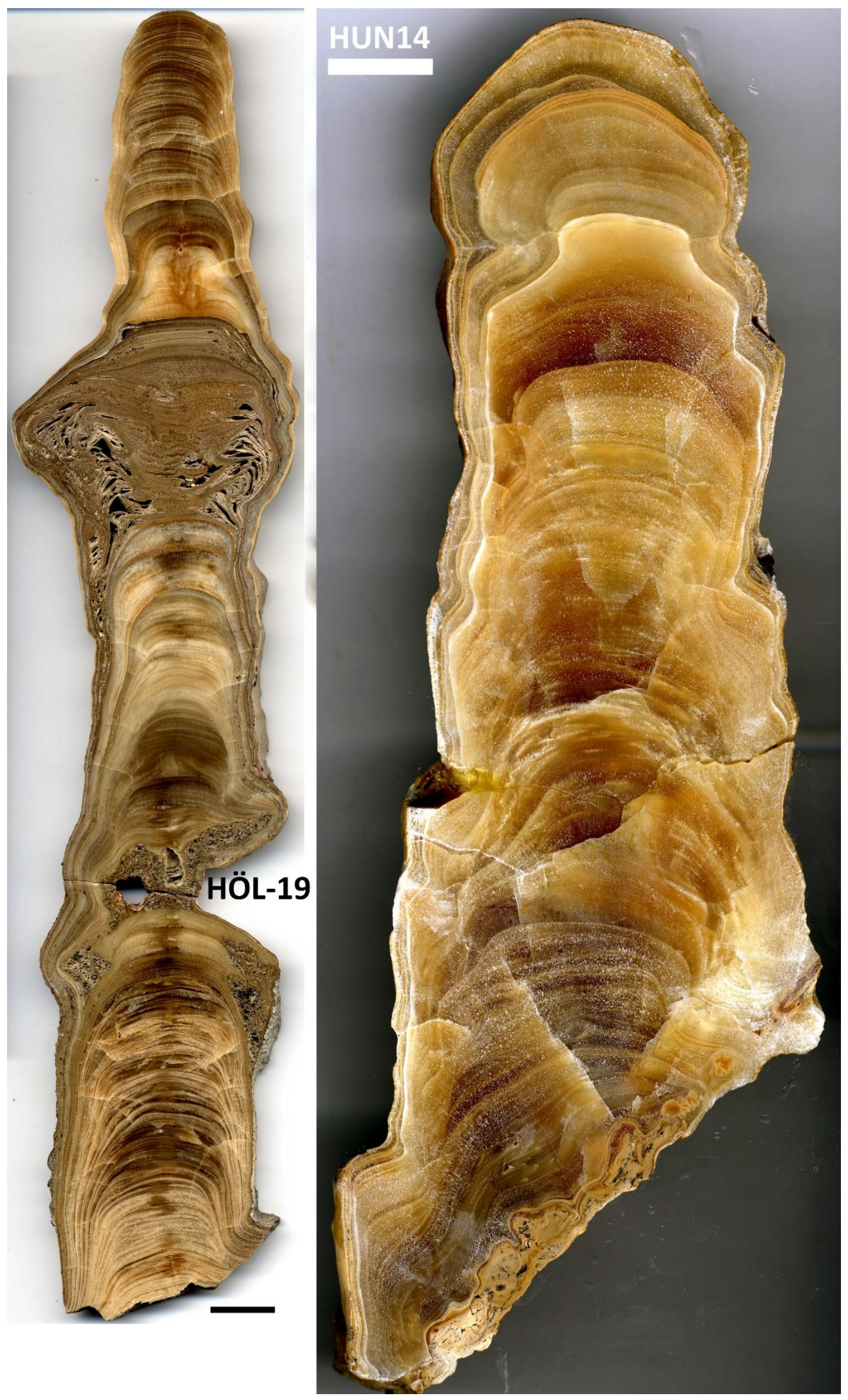

Figure S1: Samples analysed in this study. Black scale bars $=\mathbf{5 c m}$. 

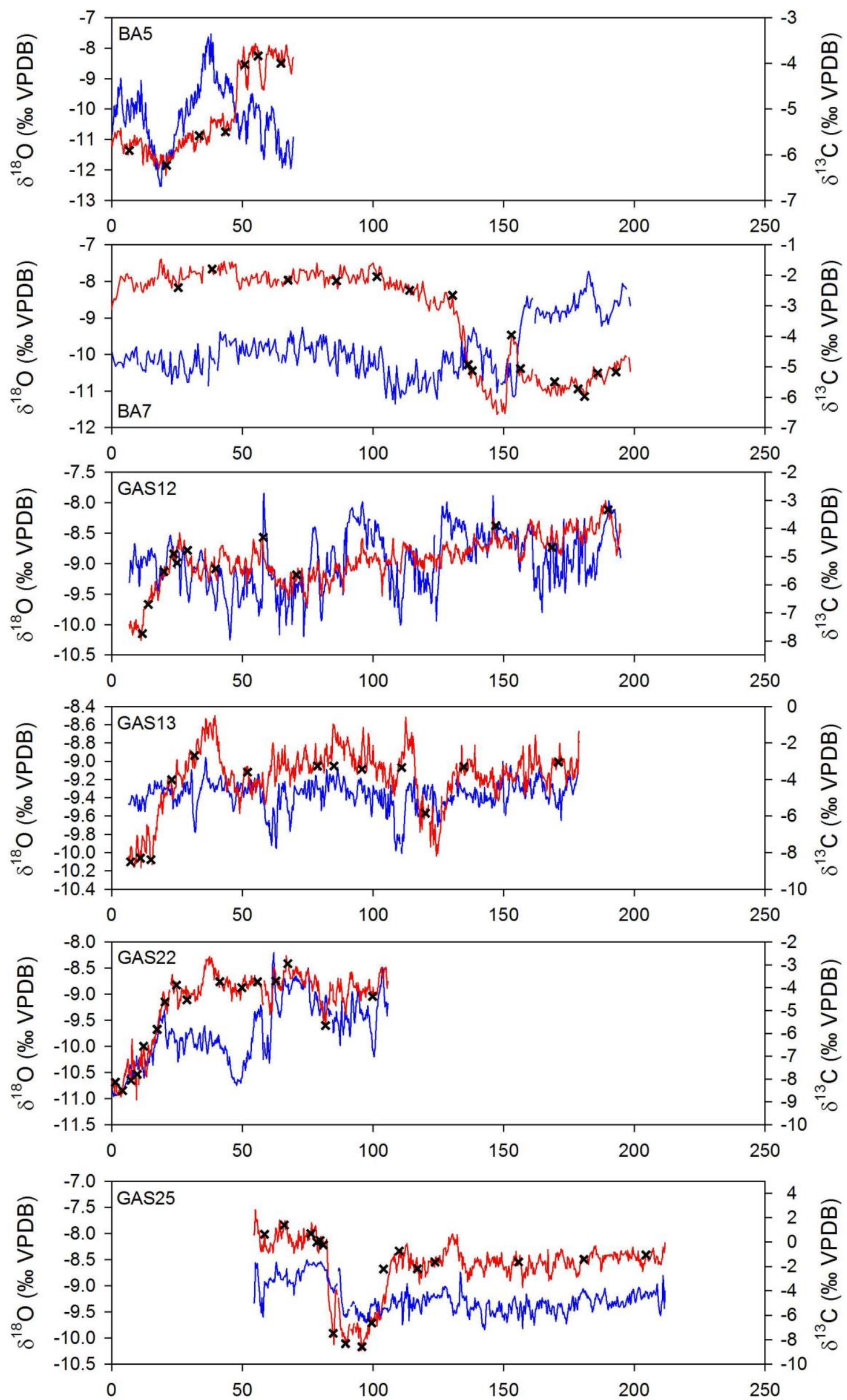

Distance along growth axis ( $\mathrm{mm}$ from top) 

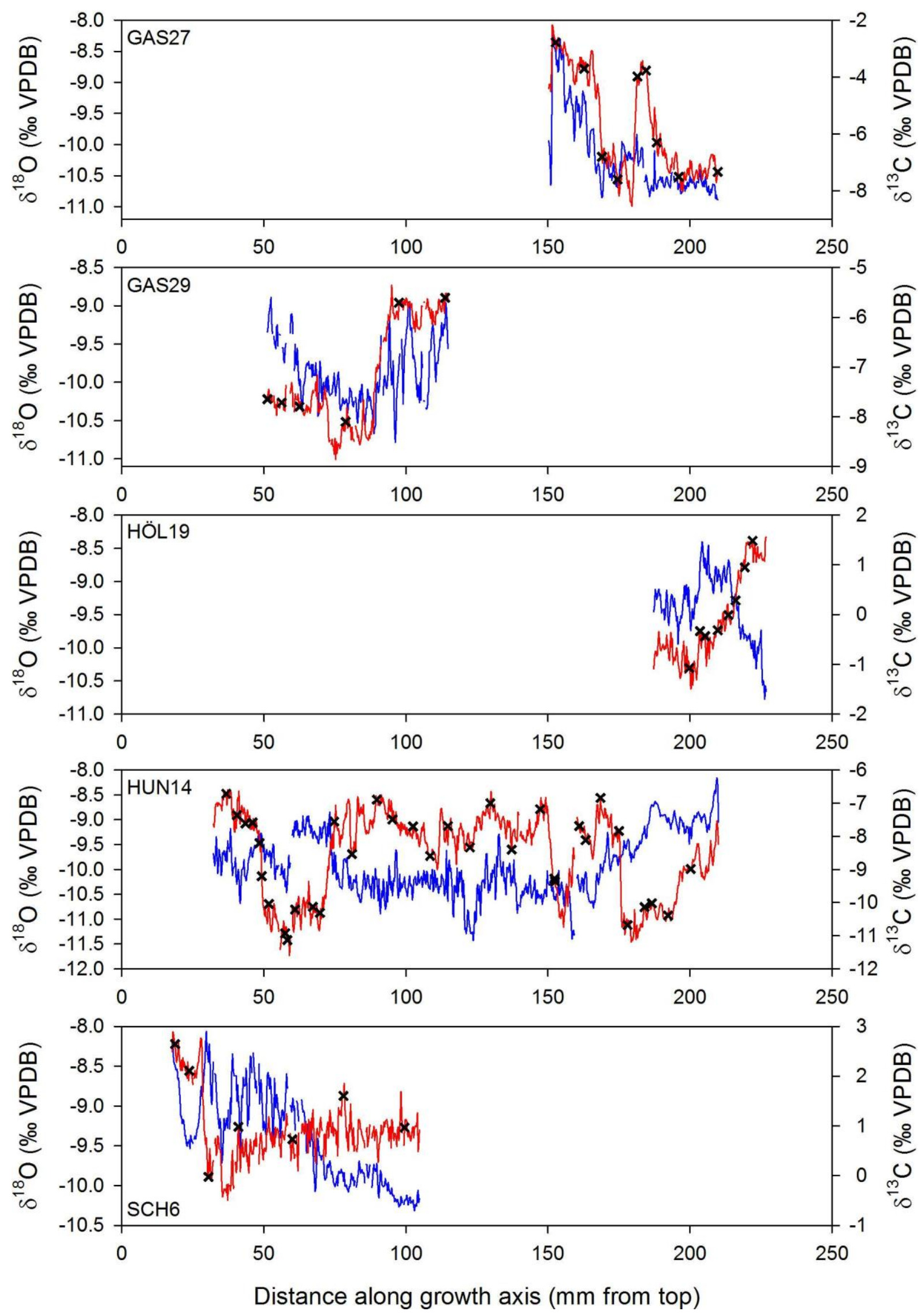

Figure S2: $\delta^{18} \mathrm{O}$ (red) and $\delta^{13} \mathrm{C}$ (blue) for each sample measured in this study relative to distance along growth axis. Black crosses mark locations of U-Th dating locations. 


\begin{tabular}{|c|c|c|c|c|c|c|c|c|}
\hline $\begin{array}{l}\text { Sample } \\
\text { (mm dft) }\end{array}$ & $\begin{array}{c}{ }^{238} \mathrm{U} \\
{\left[\mathrm{ng} \mathrm{g}^{-1}\right]}\end{array}$ & $\begin{array}{c}{ }^{232} \mathrm{Th} \\
{\left[\mathrm{pg} \mathrm{g}^{-1}\right]}\end{array}$ & $\begin{array}{l}{ }^{230} \mathrm{Th} /{ }^{232} \mathrm{Th} \\
\left(\text { atomic } \times 10^{-6} \text { ) }\right.\end{array}$ & $\begin{array}{c}\delta^{234} U^{*} \\
\text { (measured) }\end{array}$ & $\begin{array}{c}{ }^{230} \mathrm{Th} /{ }^{238} \mathrm{U} \dagger \\
\text { (activity) }\end{array}$ & $\begin{array}{c}\text { Uncorrected } \\
\text { Age }(a) \S\end{array}$ & $\begin{array}{c}\text { Corrected Age } \\
\text { (a)\# }\end{array}$ & $\begin{array}{l}\delta^{234} U^{*} \\
\text { (initial) }\end{array}$ \\
\hline \multicolumn{9}{|l|}{ BA5 } \\
\hline 6.75 & $324 \pm 0.3$ & $941 \pm 19$ & $3,940 \pm 80$ & $249 \pm 2$ & $0.6939 \pm 0.0014$ & $85,513 \pm 335$ & $85,386 \pm 337$ & $316 \pm 3$ \\
\hline 21.00 & $1085 \pm 1.0$ & $4,293 \pm 86$ & $2,900 \pm 58$ & $238 \pm 2$ & $0.6961 \pm 0.0013$ & $87,062 \pm 300$ & $86,909 \pm 306$ & $305 \pm 2$ \\
\hline 33.50 & $287 \pm 0.4$ & $726 \pm 15$ & $4,503 \pm 92$ & $228 \pm 2$ & $0.6917 \pm 0.0015$ & $87,406 \pm 368$ & $87,286 \pm 370$ & $292 \pm 3$ \\
\hline 43.50 & $295 \pm 0.4$ & $852 \pm 17$ & $3,911 \pm 79$ & $213 \pm 2$ & $0.6852 \pm 0.0014$ & $87,970 \pm 361$ & $87,841 \pm 364$ & $273 \pm 3$ \\
\hline 51.00 & $1,174 \pm 2$ & $5,261 \pm 106$ & $2,508 \pm 50$ & $200 \pm 2$ & $0.6819 \pm 0.0015$ & $88,901 \pm 405$ & $88,733 \pm 411$ & $257 \pm 3$ \\
\hline 56.00 & $312 \pm 0.4$ & $1,814 \pm 36$ & $1,943 \pm 39$ & $201 \pm 2$ & $0.6854 \pm 0.0013$ & $89,380 \pm 334$ & $89,183 \pm 346$ & $259 \pm 2$ \\
\hline 64.75 & $331 \pm 0.4$ & $1,015 \pm 20$ & $3,681 \pm 74$ & $192 \pm 2$ & $0.6838 \pm 0.0015$ & $90,195 \pm 384$ & $90,060 \pm 387$ & $248 \pm 3$ \\
\hline \multicolumn{9}{|l|}{ BA7 } \\
\hline 25.50 & $785 \pm 0.9$ & $2,764 \pm 55$ & $3,473 \pm 70$ & $365 \pm 1$ & $0.7416 \pm 0.0012$ & $81,844 \pm 230$ & $81,707 \pm 235$ & $460 \pm 2$ \\
\hline 38.50 & $378 \pm 0.4$ & $55,427 \pm 1,110$ & $85 \pm 2$ & $372 \pm 2$ & $0.7592 \pm 0.0017$ & $83,987 \pm 330$ & $80,985 \pm 2,107$ & $468 \pm 4$ \\
\hline 67.50 & $805 \pm 1.0$ & $7,239 \pm 145$ & $1,371 \pm 28$ & $364 \pm 2$ & $0.7482 \pm 0.0014$ & $83,011 \pm 289$ & $82,769 \pm 315$ & $460 \pm 3$ \\
\hline 86.00 & $718 \pm 0.8$ & $20,457 \pm 410$ & $434 \pm 9$ & $359 \pm 2$ & $0.7491 \pm 0.0012$ & $83,637 \pm 258$ & $82,999 \pm 479$ & $454 \pm 2$ \\
\hline 101.50 & $1411 \pm 1.8$ & $50,860 \pm 1,019$ & $344 \pm 7$ & $356 \pm 2$ & $0.7514 \pm 0.0015$ & $84,280 \pm 308$ & $83,491 \pm 597$ & $451 \pm 2$ \\
\hline 114.00 & $751 \pm 0.8$ & $98,543 \pm 1,973$ & $96 \pm 2$ & $349 \pm 2$ & $0.7613 \pm 0.0013$ & $86,576 \pm 267$ & $83,831 \pm 1,914$ & $443 \pm 3$ \\
\hline 130.50 & $718 \pm 1.1$ & $135,400 \pm 2,715$ & $66 \pm 1$ & $339 \pm 2$ & $0.7555 \pm 0.0017$ & $86,676 \pm 338$ & $82,705 \pm 2786$ & $428 \pm 4$ \\
\hline 136.50 & $610 \pm 0.7$ & $39,611 \pm 793$ & $190 \pm 4$ & $330 \pm 1$ & $0.7466 \pm 0.0012$ & $86,077 \pm 242$ & $84,671 \pm 977$ & $419 \pm 2$ \\
\hline 138.00 & $634 \pm 0.9$ & $62,210 \pm 1,247$ & $126 \pm 3$ & $34 \pm 2$ & $0.7526 \pm 0.0016$ & $86,711 \pm 336$ & $84,625 \pm 1469$ & $424 \pm 3$ \\
\hline 153.00 & $584 \pm 0.6$ & $34,428 \pm 689$ & $208 \pm 4$ & $327 \pm 2$ & $0.7442 \pm 0.0014$ & $85,954 \pm 279$ & $84,670 \pm 904$ & $416 \pm 2$ \\
\hline 156.50 & $566 \pm 0.6$ & $22,531 \pm 451$ & $311 \pm 6$ & $333 \pm 1$ & $0.7497 \pm 0.0012$ & $86,288 \pm 251$ & $85,405 \pm 628$ & $424 \pm 2$ \\
\hline 169.50 & $600 \pm 0.7$ & $27,111 \pm 543$ & $274 \pm 5$ & $321 \pm 2$ & $0.7492 \pm 0.0014$ & $87,421 \pm 323$ & $76,423 \pm 735$ & $410 \pm 3$ \\
\hline 178.50 & $600 \pm 0.6$ & $13,016 \pm 261$ & $567 \pm 11$ & $322 \pm 2$ & $0.7459 \pm 0.0013$ & $86,750 \pm 281$ & $86,240 \pm 423$ & $411 \pm 2$ \\
\hline 181.00 & $720 \pm 0.9$ & $9,246 \pm 185$ & $955 \pm 19$ & $320 \pm 2$ & $0.7433 \pm 0.0013$ & $86,617 \pm 280$ & $86,284 \pm 336$ & $408 \pm 2$ \\
\hline 186.00 & $507 \pm 0.6$ & $19,718 \pm 395$ & $318 \pm 6$ & $318 \pm 2$ & $0.7494 \pm 0.0014$ & $87,801 \pm 360$ & $86,932 \pm 673$ & $407 \pm 3$ \\
\hline 193.00 & $444 \pm 0.5$ & $62,846 \pm 1,259$ & $89 \pm 2$ & $310 \pm 1$ & $0.7629 \pm 0.0014$ & $91,076 \pm 303$ & $88,022 \pm 2,136$ & $398 \pm 3$ \\
\hline \multicolumn{9}{|l|}{ HÖL19 } \\
\hline 7.25 & $435 \pm 0.5$ & $29,311 \pm 587$ & $10 \pm 0.2$ & $-73 \pm 2$ & $0.0417 \pm 0.0005$ & $5,028 \pm 57$ & $3,864 \pm 591$ & $-74 \pm 2$ \\
\hline \multicolumn{9}{|l|}{ Hiatus } \\
\hline 76.00 & $467 \pm 0.4$ & $3,901 \pm 78$ & $249 \pm 5$ & $-30 \pm 1$ & $0.1261 \pm 0.0004$ & $15,195 \pm 55$ & $14,998 \pm 88$ & $-31 \pm 1$ \\
\hline 81.00 & $1,605 \pm 2$ & $1,598 \pm 32$ & $2,022 \pm 41$ & $-57 \pm 1$ & $0.1221 \pm 0.0002$ & $15,141 \pm 36$ & $15,058 \pm 37$ & $-59 \pm 1$ \\
\hline 97.25 & $985 \pm 1$ & $1,504 \pm 30$ & $1,383 \pm 28$ & $-47 \pm 1$ & $0.1280 \pm 0.0002$ & $15,748 \pm 38$ & $15,657 \pm 40$ & $-49 \pm 1$ \\
\hline \multicolumn{9}{|l|}{ Hiatus } \\
\hline 178.50 & $446 \pm 0.5$ & $1,437 \pm 29$ & $2,203 \pm 44$ & $-84 \pm 1$ & $0.4305 \pm 0.0008$ & $70,028 \pm 253$ & $69,912 \pm 255$ & $-102 \pm 2$ \\
\hline Hiatus & & & & & & & & \\
\hline \multicolumn{4}{|c|}{ ISOCHRON age for $199.75 \mathrm{~mm}$} & $-105 \pm 2$ & $0.4346 \pm 0.0014$ & $73,720 \pm 410$ & $73,656 \pm 410$ & $-129 \pm 2$ \\
\hline
\end{tabular}




\begin{tabular}{|c|c|c|c|c|c|c|c|c|}
\hline $\begin{array}{l}\text { Sample } \\
\text { (mm dft) }\end{array}$ & $\begin{array}{c}{ }^{238} \mathrm{U} \\
{\left[\mathrm{ng} \mathrm{g}^{-1}\right]}\end{array}$ & $\begin{array}{c}{ }^{232} \mathrm{Th} \\
{\left[\mathrm{pg} \mathrm{g}^{-1}\right]}\end{array}$ & $\begin{array}{l}{ }^{230} \mathrm{Th} /{ }^{232} \mathrm{Th} \\
\text { (atomic } \times 10^{-6} \text { ) }\end{array}$ & $\begin{array}{c}\delta^{234} U^{*} \\
\text { (measured) }\end{array}$ & $\begin{array}{c}{ }^{230} \text { Th / }{ }^{238} \mathrm{U}+ \\
\text { (activity) }\end{array}$ & $\begin{array}{c}\text { Uncorrected } \\
\text { Age }(a) \S\end{array}$ & $\begin{array}{c}\text { Corrected Age } \\
\text { (a)\# }\end{array}$ & $\begin{array}{l}\delta^{234} U^{*} \\
\text { (initial) }\end{array}$ \\
\hline \multicolumn{9}{|l|}{ HÖL19 } \\
\hline 203.50 & $777 \pm 1$ & $2,736 \pm 55$ & $2,034 \pm 41$ & $-108 \pm 1$ & $0.4346 \pm 0.0009$ & $74,156 \pm 300$ & $74,032 \pm 302$ & $-134 \pm 2$ \\
\hline 205.25 & $822 \pm 1$ & $1,964 \pm 39$ & $2,982 \pm 60$ & $-113 \pm 1$ & $0.4322 \pm 0.0010$ & $74,140 \pm 292$ & $74,031 \pm 292$ & $-139 \pm 2$ \\
\hline 209.75 & $759 \pm 1$ & $4,104 \pm 82$ & $1,327 \pm 27$ & $-108 \pm 1$ & $0.4348 \pm 0.0009$ & $74,127 \pm 298$ & $73,970 \pm 301$ & $-133 \pm 2$ \\
\hline 213.50 & $603 \pm 1$ & $5,058 \pm 101$ & $861 \pm 17$ & $-106 \pm 1$ & $0.4381 \pm 0.0010$ & $74,671 \pm 315$ & $74,459 \pm 324$ & $-131 \pm 2$ \\
\hline 216.00 & $492 \pm 1$ & $4,260 \pm 85$ & $832 \pm 17$ & $-104 \pm 1$ & $0.4368 \pm 0.0009$ & $74,072 \pm 290$ & $73,859 \pm 300$ & $-128 \pm 2$ \\
\hline 219.25 & $674 \pm 1$ & $5,186 \pm 104$ & $939 \pm 19$ & $-106 \pm 2$ & $0.4379 \pm 0.0012$ & $74,638 \pm 373$ & $74,441 \pm 379$ & $-131 \pm 2$ \\
\hline 222.00 & $648 \pm 1$ & $2,057 \pm 41$ & $2,261 \pm 45$ & $-109 \pm 1$ & $0.4356 \pm 0.0009$ & $74,426 \pm 284$ & $74,303 \pm 285$ & $-134 \pm 2$ \\
\hline \multicolumn{9}{|l|}{ Hiatus } \\
\hline 394.00 & $851 \pm 1$ & $54,302 \pm 1,088$ & $128 \pm 3$ & $-61 \pm 2$ & $0.4959 \pm 0.0011$ & $82,747 \pm 429$ & $81,638 \pm 700$ & $-77 \pm 3$ \\
\hline \multicolumn{9}{|l|}{$\underline{\mathrm{SCH} 6}$} \\
\hline 11.25 & $166 \pm 0.2$ & $27,618 \pm 553$ & $70 \pm 1$ & $382 \pm 2$ & $0.7071 \pm 0.0035$ & $75,074 \pm 529$ & $71,664 \pm 2,424$ & $468 \pm 4$ \\
\hline \multicolumn{9}{|l|}{ Hiatus } \\
\hline 18.75 & $54 \pm 0.04$ & $2,294 \pm 46$ & $301 \pm 6$ & $493 \pm 2$ & $0.7689 \pm 0.0020$ & $75,275 \pm 304$ & $74,443 \pm 520$ & $608 \pm 3$ \\
\hline 23.75 & $66 \pm 0.05$ & $1,979 \pm 40$ & $428 \pm 9$ & $499 \pm 2$ & $0.7785 \pm 0.0017$ & $76,077 \pm 263$ & $75,469 \pm 463$ & $618 \pm 2$ \\
\hline 30.50 & $166 \pm 0.11$ & $584 \pm 12$ & $3,757 \pm 76$ & $543 \pm 1$ & $0.8022 \pm 0.0014$ & $76,024 \pm 214$ & $75,896 \pm 218$ & $673 \pm 2$ \\
\hline 41.00 & $269 \pm 0.30$ & $267 \pm 5$ & $13,477 \pm 278$ & $551 \pm 2$ & $0.8110 \pm 0.0012$ & $76,544 \pm 211$ & $76,460 \pm 211$ & $684 \pm 2$ \\
\hline 60.00 & $259 \pm 0.24$ & $793 \pm 16$ & $4,375 \pm 88$ & $548 \pm 2$ & $0.8131 \pm 0.0012$ & $77,081 \pm 198$ & $76,961 \pm 202$ & $681 \pm 2$ \\
\hline 78.00 & $209 \pm 0.20$ & $502 \pm 10$ & $5,594 \pm 113$ & $546 \pm 2$ & $0.8145 \pm 0.0013$ & $77,430 \pm 220$ & $77,321 \pm 222$ & $679 \pm 2$ \\
\hline 99.50 & $222 \pm 0.21$ & $575 \pm 12$ & $5,182 \pm 105$ & $538 \pm 2$ & $0.8148 \pm 0.0012$ & $78,038 \pm 204$ & $77,926 \pm 206$ & $670 \pm 2$ \\
\hline \multicolumn{9}{|l|}{ HUN14 } \\
\hline 36.75 & $576 \pm 0.6$ & $1,126 \pm 23$ & $6,842 \pm 138$ & $288 \pm 1$ & $0.8107 \pm 0.0011$ & $102,942 \pm 297$ & $102,834 \pm 298$ & $385 \pm 2$ \\
\hline 40.50 & $673 \pm 2$ & $1,279 \pm 7$ & $6,854 \pm 36$ & $255 \pm 1$ & $0.7899 \pm 0.0012$ & $103,443 \pm 312$ & $103,335 \pm 313$ & $341 \pm 2$ \\
\hline 43.50 & $534 \pm 0.6$ & $1,280 \pm 26$ & $5,446 \pm 110$ & $256 \pm 2$ & $0.7921 \pm 0.0014$ & $103,770 \pm 402$ & $103,654 \pm 404$ & $343 \pm 2$ \\
\hline 45.50 & $389 \pm 0.4$ & $1,455 \pm 29$ & $3,426 \pm 69$ & $233 \pm 2$ & $0.7774 \pm 0.0013$ & $104,030 \pm 376$ & $103,883 \pm 380$ & $312 \pm 2$ \\
\hline 48.50 & $468 \pm 1$ & $635 \pm 4$ & $9,156 \pm 47$ & $201 \pm 1$ & $0.7537 \pm 0.0007$ & $103,732 \pm 242$ & $103,634 \pm 243$ & $269 \pm 2$ \\
\hline 49.25 & $365 \pm 0.3$ & $873 \pm 18$ & $5,278 \pm 107$ & $187 \pm 1$ & $0.7457 \pm 0.0010$ & $104,093 \pm 326$ & $103,971 \pm 328$ & $251 \pm 2$ \\
\hline 51.75 & $413 \pm 0.5$ & $512 \pm 10$ & $110,443 \pm 212$ & $243 \pm 2$ & $0.7849 \pm 0.0014$ & $104,095 \pm 391$ & $104,004 \pm 391$ & $326 \pm 2$ \\
\hline 57.50 & $555 \pm 1$ & $479 \pm 3$ & $14,456 \pm 77$ & $205 \pm 1$ & $0.7570 \pm 0.0008$ & $103,803 \pm 298$ & $103,717 \pm 299$ & $275 \pm 2$ \\
\hline 58.25 & $536 \pm 0.5$ & $301 \pm 6$ & $22,112 \pm 462$ & $195 \pm 1$ & $0.7526 \pm 0.0010$ & $104,357 \pm 310$ & $104,277 \pm 310$ & $262 \pm 2$ \\
\hline \multicolumn{9}{|l|}{ Hiatus } \\
\hline 61.00 & $315 \pm 0.3$ & $787 \pm 16$ & $4910 \pm 101$ & $174 \pm 2$ & $0.7440 \pm 0.0016$ & $105,787 \pm 490$ & $105,664 \pm 491$ & $235 \pm 3$ \\
\hline 67.50 & $250 \pm 0.2$ & $413 \pm 8$ & $7893 \pm 161$ & $241 \pm 2$ & $0.7909 \pm 0.0012$ & $105,718 \pm 345$ & $105,617 \pm 346$ & $325 \pm 2$ \\
\hline 69.75 & $349 \pm 0.3$ & $547 \pm 11$ & $8,612 \pm 174$ & $282 \pm 1$ & $0.8201 \pm 0.0010$ & $105,732 \pm 301$ & $105,632 \pm 302$ & $380 \pm 2$ \\
\hline 74.75 & $494 \pm 0.6$ & $266 \pm 5$ & $25,249 \pm 522$ & $286 \pm 2$ & $0.8232 \pm 0.0015$ & $105,895 \pm 391$ & $105,820 \pm 391$ & $385 \pm 2$ \\
\hline
\end{tabular}




\begin{tabular}{|c|c|c|c|c|c|c|c|c|}
\hline $\begin{array}{l}\text { Sample } \\
\text { (mm dft) }\end{array}$ & $\begin{array}{c}{ }^{238} \mathrm{U} \\
{\left[\mathrm{ng} \mathrm{g}^{-1}\right]}\end{array}$ & $\begin{array}{c}{ }^{232} \mathrm{Th} \\
{\left[\mathrm{pg} \mathrm{g}^{-1}\right]}\end{array}$ & $\begin{array}{l}{ }^{230} \mathrm{Th} /{ }^{232} \mathrm{Th} \\
\left(\text { atomic } \times 10^{-6} \text { ) }\right.\end{array}$ & $\begin{array}{c}\delta^{234} U^{*} \\
\text { (measured) }\end{array}$ & $\begin{array}{c}{ }^{230} \mathrm{Th} /{ }^{238} \mathrm{U}+ \\
\text { (activity) }\end{array}$ & $\begin{array}{c}\text { Uncorrected } \\
\text { Age }(a) \S\end{array}$ & $\begin{array}{c}\text { Corrected Age } \\
\text { (a)\# }\end{array}$ & $\begin{array}{l}\delta^{234} U^{*} \\
\text { (initial) }\end{array}$ \\
\hline \multicolumn{9}{|l|}{ HUN14 } \\
\hline 81.00 & $455 \pm 0.6$ & $1,146 \pm 23$ & $5,595 \pm 113$ & $327 \pm 2$ & $0.8553 \pm 0.0016$ & $106,486 \pm 402$ & $106,371 \pm 404$ & $441 \pm 2$ \\
\hline 89.75 & $570 \pm 0.7$ & $296 \pm 7$ & $27,371 \pm 615$ & $339 \pm 2$ & $0.8624 \pm 0.0017$ & $106,231 \pm 442$ & $106,156 \pm 442$ & $457 \pm 3$ \\
\hline 95.25 & $720 \pm 2$ & $446 \pm 3$ & $22,289 \pm 120$ & $301 \pm 2$ & $0.8366 \pm 0.0010$ & $106,473 \pm 316$ & $106,393 \pm 315$ & $406 \pm 2$ \\
\hline 102.50 & $957 \pm 1$ & $153 \pm 4$ & $84,773 \pm 1,949$ & $283 \pm 2$ & $0.8238 \pm 0.0013$ & $106,391 \pm 381$ & $106,320 \pm 381$ & $382 \pm 2$ \\
\hline 108.50 & $449 \pm 0.5$ & $127 \pm 3$ & $48,120 \pm 1,121$ & $277 \pm 2$ & $0.8232 \pm 0.0014$ & $107,081 \pm 374$ & $107,010 \pm 374$ & $375 \pm 2$ \\
\hline 115.00 & $612 \pm 0.6$ & $250 \pm 5$ & $33,598 \pm 708$ & $289 \pm 1$ & $0.8313 \pm 0.0013$ & $107,121 \pm 343$ & $107,049 \pm 343$ & $391 \pm 2$ \\
\hline 122.50 & $824 \pm 2$ & $331 \pm 2$ & $33,927 \pm 192$ & $282 \pm 2$ & $0.8261 \pm 0.0011$ & $106,950 \pm 353$ & $106,874 \pm 353$ & $382 \pm 2$ \\
\hline 129.75 & $407 \pm 0.4$ & $117 \pm 3$ & $47,557 \pm 1,086$ & $281 \pm 2$ & $0.8273 \pm 0.0013$ & $107,420 \pm 366$ & $107,350 \pm 366$ & $381 \pm 2$ \\
\hline 137.25 & $560 \pm 0.4$ & $695 \pm 14$ & $10,684 \pm 216$ & $250 \pm 1$ & $0.8047 \pm 0.0010$ & $107,297 \pm 315$ & $107,202 \pm 316$ & $339 \pm 2$ \\
\hline 147.25 & $622 \pm 0.6$ & $97 \pm 2$ & $82,601 \pm 1,299$ & $213 \pm 1$ & $0.7772 \pm 0.0013$ & $107,138 \pm 375$ & $107,071 \pm 375$ & $288 \pm 2$ \\
\hline 152.25 & $742 \pm 0.7$ & $230 \pm 1$ & $40,716 \pm 224$ & $192 \pm 1$ & $0.7642 \pm 0.0011$ & $107,585 \pm 331$ & $107,514 \pm 331$ & $260 \pm 2$ \\
\hline 152.50 & $524 \pm 0.5$ & $324 \pm 7$ & $20,374 \pm 425$ & $191 \pm 1$ & $0.7633 \pm 0.0010$ & $107,415 \pm 338$ & $107,333 \pm 338$ & $259 \pm 2$ \\
\hline \multicolumn{9}{|l|}{ Hiatus } \\
\hline 161.00 & $708 \pm 2$ & $1,336 \pm 27$ & $6,760 \pm 139$ & $203 \pm 2$ & $0.7733 \pm 0.0026$ & $107,867 \pm 910$ & $107,757 \pm 910$ & $275 \pm 3$ \\
\hline 163.25 & $692 \pm 0.5$ & $1,051 \pm 21$ & $8,375 \pm 169$ & $200 \pm 1$ & $0.7719 \pm 0.0009$ & $108,107 \pm 309$ & $108,004 \pm 310$ & $270 \pm 2$ \\
\hline 168.50 & $519 \pm 0.3$ & $2,001 \pm 40$ & $3,299 \pm 66$ & $195 \pm 1$ & $0.7707 \pm 0.0010$ & $108,513 \pm 315$ & $108,356 \pm 321$ & $265 \pm 2$ \\
\hline 175.00 & $572 \pm 1$ & $1,244 \pm 25$ & $5,644 \pm 115$ & $157 \pm 2$ & $0.7442 \pm 0.0025$ & $108,796 \pm 702$ & $108,676 \pm 703$ & $213 \pm 3$ \\
\hline 178.00 & $1,398 \pm 1$ & $1,542 \pm 31$ & $11,118 \pm 223$ & $156 \pm 1$ & $0.7435 \pm 0.0011$ & $108,818 \pm 361$ & $108,727 \pm 361$ & $212 \pm 2$ \\
\hline 184.00 & $769 \pm 1$ & $764 \pm 16$ & $12,389 \pm 254$ & $157 \pm 2$ & $0.7469 \pm 0.0016$ & $109,375 \pm 487$ & $109,287 \pm 488$ & $214 \pm 2$ \\
\hline 186.50 & $786 \pm 2$ & $1,846 \pm 37$ & $5477 \pm 111$ & $200 \pm 2$ & $0.7808 \pm 0.0023$ & $110,128 \pm 647$ & $110,007 \pm 648$ & $272 \pm 3$ \\
\hline 192.25 & $820 \pm 0.8$ & $725 \pm 15$ & $15,299 \pm 309$ & $252 \pm 2$ & $0.8201 \pm 0.0012$ & $110,453 \pm 367$ & $110,370 \pm 367$ & $344 \pm 2$ \\
\hline 200.25 & $1,307 \pm 1$ & $1,468 \pm 29$ & $12,456 \pm 251$ & $290 \pm 2$ & $0.8490 \pm 0.0013$ & $110,643 \pm 378$ & $110,556 \pm 378$ & $397 \pm 2$ \\
\hline \multicolumn{9}{|l|}{ GAS12 } \\
\hline 11.75 & $344 \pm 0.7$ & $21 \pm 6$ & $152,718 \pm 48,200$ & $82 \pm 3$ & $0.5539 \pm 0.0029$ & $77,363 \pm 659$ & $77,303 \pm 659$ & $102 \pm 3$ \\
\hline 14.00 & $389 \pm 0.5$ & $26 \pm 2$ & $139,450 \pm 8,216$ & $87 \pm 2$ & $05561 \pm 0.0011$ & $77,151 \pm 285$ & $77,085 \pm 285$ & $109 \pm 2$ \\
\hline 20.00 & $461 \pm 0.5$ & $22 \pm 2$ & $192,533 \pm 13,954$ & $86 \pm 1$ & $0.5568 \pm 0.0012$ & $77,480 \pm 283$ & $77,414 \pm 283$ & $107 \pm 2$ \\
\hline 23.75 & $433 \pm 0.4$ & $10 \pm 1$ & $396,030 \pm 45084$ & $87 \pm 1$ & $0.5571 \pm 0.0008$ & $77,443 \pm 210$ & $77,380 \pm 210$ & $108 \pm 2$ \\
\hline 25.00 & $453 \pm 0.6$ & $24 \pm 1$ & $171,066 \pm 9,163$ & $87 \pm 2$ & $0.5571 \pm 0.0014$ & $77,457 \pm 330$ & $77,391 \pm 330$ & $108 \pm 2$ \\
\hline 29.00 & $316 \pm 0.3$ & $42 \pm 2$ & $69,414 \pm 3,290$ & $91 \pm 2$ & $0.5602 \pm 0.0014$ & $77,605 \pm 335$ & $77,537 \pm 335$ & $113 \pm 2$ \\
\hline 39.75 & $384 \pm 0.3$ & $31 \pm 1$ & $117,230 \pm 5263$ & $96 \pm 1$ & $0.5654 \pm 0.0008$ & $78,082 \pm 224$ & $78,018 \pm 224$ & $119 \pm 2$ \\
\hline 58.00 & $209 \pm 0.3$ & $194 \pm 4$ & $10,101 \pm 223$ & $97 \pm 2$ & $0.5683 \pm 0.0025$ & $78,543 \pm 536$ & $78,456 \pm 537$ & $121 \pm 2$ \\
\hline 70.75 & $463 \pm 0.5$ & $53 \pm 2$ & $82,524 \pm 2,504$ & $109 \pm 1$ & $0.5780 \pm 0.0008$ & $79,125 \pm 230$ & $79,060 \pm 230$ & $136 \pm 2$ \\
\hline 147.00 & $211 \pm 0.3$ & $30 \pm 2$ & $68,306 \pm 3,955$ & $120 \pm 2$ & $0.5937 \pm 0.0019$ & $80,959 \pm 417$ & $80,892 \pm 417$ & $151 \pm 2$ \\
\hline 168.50 & $370 \pm 0.3$ & $76 \pm 2$ & $48,569 \pm 1,244$ & $134 \pm 1$ & $0.6024 \pm 0.0009$ & $81,108 \pm 234$ & $81,041 \pm 234$ & $168 \pm 2$ \\
\hline
\end{tabular}




\begin{tabular}{|c|c|c|c|c|c|c|c|c|}
\hline $\begin{array}{l}\text { Sample } \\
\text { (mm dft) }\end{array}$ & $\begin{array}{c}{ }^{238} \mathrm{U} \\
{\left[\mathrm{ng} \mathrm{g}^{-1}\right]}\end{array}$ & $\begin{array}{c}{ }^{232} \mathrm{Th} \\
{\left[\mathrm{pg} \mathrm{g}^{-1}\right]}\end{array}$ & $\begin{array}{l}{ }^{230} \mathrm{Th} /{ }^{232} \mathrm{Th} \\
\left(\text { atomic } \times 10^{-6} \text { ) }\right.\end{array}$ & $\begin{array}{c}\delta^{234} U^{*} \\
\text { (measured) }\end{array}$ & $\begin{array}{c}{ }^{230} \mathrm{Th} /{ }^{238} \mathrm{U} \dagger \\
\text { (activity) }\end{array}$ & $\begin{array}{c}\text { Uncorrected } \\
\text { Age (a)§ }\end{array}$ & $\begin{array}{c}\text { Corrected Age } \\
\text { (a)\# }\end{array}$ & $\begin{array}{l}\delta^{234} U^{*} \\
\text { (initial) }\end{array}$ \\
\hline \multicolumn{9}{|l|}{ GAS12 } \\
\hline 190.00 & $192 \pm 0.2$ & $54 \pm 2$ & $35,131 \pm 1,279$ & $120 \pm 2$ & $0.5992 \pm 0.0019$ & $81,999 \pm 426$ & $81,929 \pm 426$ & $152 \pm 2$ \\
\hline \multicolumn{9}{|l|}{ GAS13 } \\
\hline 7.25 & $118 \pm 0.1$ & $143 \pm 3$ & $7,280 \pm 170$ & $52 \pm 1$ & $0.5344 \pm 0.0024$ & $76,815 \pm 523$ & $76,719 \pm 523$ & $64 \pm 2$ \\
\hline 11.00 & $247 \pm 0.2$ & $88 \pm 2$ & $24,815 \pm 682$ & $51 \pm 1$ & $0.5350 \pm 0.0011$ & $77,028 \pm 280$ & $76,954 \pm 280$ & $63 \pm 2$ \\
\hline 15.00 & $383 \pm 0.5$ & $42 \pm 1$ & $79,942 \pm 1,932$ & $46 \pm 2$ & $0.5329 \pm 0.0011$ & $77,202 \pm 288$ & $77,138 \pm 288$ & $57 \pm 2$ \\
\hline 23.00 & $418 \pm 0.4$ & $57 \pm 2$ & $64,474 \pm 1,851$ & $45 \pm 1$ & $0.5340 \pm 0.0009$ & $77,469 \pm 250$ & $77,401 \pm 250$ & $56 \pm 2$ \\
\hline 31.75 & $419 \pm 0.4$ & $96 \pm 3$ & $38,304 \pm 1,098$ & $46 \pm 1$ & $0.5343 \pm 0.0008$ & $77,446 \pm 230$ & $77,376 \pm 230$ & $57 \pm 2$ \\
\hline 52.00 & $442 \pm 0.4$ & $28 \pm 2$ & $141,138 \pm 7,662$ & $47 \pm 1$ & $0.5369 \pm 0.0009$ & $77,851 \pm 241$ & $77,785 \pm 241$ & $59 \pm 2$ \\
\hline 78.75 & $373 \pm 0.3$ & $96 \pm 3$ & $34,570 \pm 911$ & $51 \pm 1$ & $0.5409 \pm 0.0008$ & $78,248 \pm 238$ & $78,178 \pm 238$ & $64 \pm 2$ \\
\hline 85.00 & $232 \pm 0.2$ & $20 \pm 2$ & $104,163 \pm 10,256$ & $49 \pm 1$ & $0.5405 \pm 0.0015$ & $78,433 \pm 354$ & $78,367 \pm 354$ & $61 \pm 2$ \\
\hline 95.75 & $409 \pm 0.4$ & $239 \pm 5$ & $15,285 \pm 323$ & $50 \pm 1$ & $0.5414 \pm 0.0008$ & $78,494 \pm 239$ & $78,415 \pm 240$ & $62 \pm 2$ \\
\hline 111.00 & $490 \pm 0.6$ & $44 \pm 2$ & $99,706 \pm 5,094$ & $49 \pm 2$ & $0.5406 \pm 0.0011$ & $78,484 \pm 292$ & $78,417 \pm 292$ & $61 \pm 2$ \\
\hline 120.00 & $442 \pm 0.4$ & $37 \pm 2$ & $105,776 \pm 5,544$ & $49 \pm 1$ & $0.5426 \pm 0.0009$ & $78,856 \pm 259$ & $78,790 \pm 259$ & $61 \pm 2$ \\
\hline 134.75 & $456 \pm 0.5$ & $18 \pm 2$ & $227,539 \pm 24,237$ & $50 \pm 2$ & $0.5449 \pm 0.0011$ & $79,231 \pm 294$ & $79,168 \pm 294$ & $62 \pm 2$ \\
\hline 171.00 & $488 \pm 0.5$ & $333 \pm 7$ & $13,320 \pm 269$ & $50 \pm 1$ & $0.5506 \pm 0.0009$ & $80,380 \pm 265$ & $80,300 \pm 266$ & $63 \pm 2$ \\
\hline \multicolumn{9}{|l|}{ GAS22 } \\
\hline 1.4 & $322 \pm 0.3$ & $150 \pm 4$ & $24,772 \pm 591$ & $108 \pm 1$ & $0.6975 \pm 0.0011$ & $105,810 \pm 378$ & $105,731 \pm 378$ & $146 \pm 2$ \\
\hline 4.2 & $297 \pm 1.3$ & $62 \pm 0.4$ & $54,315 \pm 290$ & $103 \pm 1$ & $0.6921 \pm 0.0009$ & $105,371 \pm 355$ & $105,299 \pm 355$ & $139 \pm 2$ \\
\hline 7.4 & $305 \pm 1$ & $17 \pm 0.1$ & $205,514 \pm 1,212$ & $103 \pm 2$ & $0.6916 \pm 0.0009$ & $105,227 \pm 376$ & $105,159 \pm 376$ & $139 \pm 2$ \\
\hline 9.8 & $337 \pm 0.4$ & $16 \pm 1$ & $242,747 \pm 14,749$ & $102 \pm 1$ & $0.6915 \pm 0.0011$ & $105,313 \pm 378$ & $105,244 \pm 378$ & $138 \pm 2$ \\
\hline 12.2 & $317 \pm 2$ & $16 \pm 0.1$ & $221,352 \pm 1,371$ & $104 \pm 2$ & $0.6911 \pm 0.0011$ & $104,932 \pm 404$ & $104,864 \pm 404$ & $140 \pm 2$ \\
\hline 17.4 & $325 \pm 0.4$ & $19 \pm 1$ & $195,158 \pm 10,918$ & $102 \pm 2$ & $0.6945 \pm 0.0010$ & $106,247 \pm 369$ & $106,178 \pm 369$ & $137 \pm 2$ \\
\hline 20.4 & $262 \pm 1$ & $19 \pm 0.1$ & $158,654 \pm 943$ & $100 \pm 2$ & $0.6927 \pm 0.0010$ & $105,998 \pm 378$ & $105,929 \pm 378$ & $135 \pm 2$ \\
\hline 24.8 & $311 \pm 1$ & $13 \pm 0.1$ & $274,463 \pm 1,776$ & $99 \pm 2$ & $0.6922 \pm 0.0010$ & $106,056 \pm 380$ & $105,988 \pm 380$ & $134 \pm 2$ \\
\hline 28.8 & $340 \pm 0.3$ & $39 \pm 1$ & $99,845 \pm 3,321$ & $100 \pm 1$ & $0.6933 \pm 0.0009$ & $106,145 \pm 324$ & $106,075 \pm 324$ & $135 \pm 2$ \\
\hline 41.1 & $353 \pm 0.5$ & $10 \pm 1$ & $419,777 \pm 60,479$ & $101 \pm 2$ & $0.6954 \pm 0.0018$ & $106,636 \pm 575$ & $106,573 \pm 575$ & $136 \pm 3$ \\
\hline 49.8 & $172 \pm 0.2$ & $13 \pm 2$ & $155,310 \pm 20,730$ & $102 \pm 2$ & $0.6963 \pm 0.0023$ & $106,552 \pm 673$ & $106,487 \pm 673$ & $138 \pm 2$ \\
\hline 55.8 & $321 \pm 0.3$ & $29 \pm 4$ & $125,513 \pm 16,346$ & $100 \pm 1$ & $0.6985 \pm 0.0014$ & $107,556 \pm 430$ & $107,487 \pm 430$ & $135 \pm 2$ \\
\hline 62.8 & $347 \pm 0.4$ & $89 \pm 3$ & $44,715 \pm 1,450$ & $99 \pm 1$ & $0.6946 \pm 0.0011$ & $106,677 \pm 380$ & $106,603 \pm 380$ & $134 \pm 2$ \\
\hline 67.4 & $352 \pm 0.3$ & $26 \pm 2$ & $157,626 \pm 9,298$ & $100 \pm 1$ & $0.6974 \pm 0.0011$ & $107,352 \pm 380$ & $107,286 \pm 381$ & $135 \pm 2$ \\
\hline 81.8 & $339 \pm 0.3$ & $12 \pm 2$ & $328,531 \pm 56,430$ & $96 \pm 1$ & $0.6954 \pm 0.0012$ & $107,565 \pm 368$ & $107,497 \pm 368$ & $129 \pm 2$ \\
\hline 99.8 & $309 \pm 0.3$ & $61 \pm 2$ & $58,409 \pm 1,656$ & $106 \pm 1$ & $0.7038 \pm 0.0010$ & $107,827 \pm 355$ & $107,758 \pm 355$ & $144 \pm 2$ \\
\hline \multicolumn{9}{|l|}{ GAS25 } \\
\hline 58.50 & $295 \pm 0.3$ & $455 \pm 9$ & $6132 \pm 125$ & $61 \pm 2$ & $0.5737 \pm 0.0011$ & $84,045 \pm 339$ & $83,942 \pm 340$ & $77 \pm 2$ \\
\hline
\end{tabular}




\begin{tabular}{|c|c|c|c|c|c|c|c|c|}
\hline $\begin{array}{l}\text { Sample } \\
\text { (mm dft) }\end{array}$ & $\begin{array}{c}{ }^{238} \mathrm{U} \\
{\left[\mathrm{ng} \mathrm{g}^{-1}\right]}\end{array}$ & $\begin{array}{c}{ }^{232} \mathrm{Th} \\
{\left[\mathrm{pg} \mathrm{g}^{-1}\right]}\end{array}$ & $\begin{array}{l}{ }^{230} \mathrm{Th} /{ }^{232} \mathrm{Th} \\
\text { (atomic } \times 10^{-6} \text { ) }\end{array}$ & $\begin{array}{c}\delta^{234} U^{*} \\
\text { (measured) }\end{array}$ & $\begin{array}{c}{ }^{230} \mathrm{Th} /{ }^{238} \mathrm{U}+ \\
\text { (activity) }\end{array}$ & $\begin{array}{c}\text { Uncorrected } \\
\text { Age }(a) \S\end{array}$ & $\begin{array}{c}\text { Corrected Age } \\
\text { (a)\# }\end{array}$ & $\begin{array}{l}\delta^{234} U^{*} \\
\text { (initial) }\end{array}$ \\
\hline \multicolumn{9}{|l|}{ GAS25 } \\
\hline 66.00 & $401 \pm 0.4$ & $27 \pm 2$ & $141,308 \pm 7,990$ & $61 \pm 1$ & $0.5752 \pm 0.0010$ & $84,383 \pm 284$ & $84,317 \pm 284$ & $77 \pm 2$ \\
\hline 76.25 & $315 \pm 0.3$ & $52 \pm 2$ & $58,083 \pm 1,936$ & $63 \pm 1$ & $0.5769 \pm 0.0010$ & $84,518 \pm 285$ & $84,450 \pm 285$ & $80 \pm 2$ \\
\hline 78.50 & $315 \pm 0.4$ & $23 \pm 1$ & $129,751 \pm 7,210$ & $60 \pm 2$ & $0.5759 \pm 0.0012$ & $84,592 \pm 356$ & $84,528 \pm 356$ & $77 \pm 2$ \\
\hline 79.75 & $299 \pm 0.3$ & $35 \pm 2$ & $81,909 \pm 3,797$ & $66 \pm 2$ & $0.5785 \pm 0.0016$ & $84,426 \pm 427$ & $84,359 \pm 427$ & $84 \pm 2$ \\
\hline 81.00 & $276 \pm 0.3$ & $59 \pm 2$ & $45,241 \pm 1,561$ & $69 \pm 1$ & $0.5816 \pm 0.0010$ & $84,714 \pm 296$ & $84,644 \pm 296$ & $88 \pm 2$ \\
\hline 84.75 & $249 \pm 0.2$ & $91 \pm 2$ & $26,228 \pm 667$ & $69 \pm 2$ & $0.5823 \pm 0.0014$ & $84,801 \pm 380$ & $84,728 \pm 380$ & $88 \pm 2$ \\
\hline \multicolumn{9}{|l|}{ Hiatus } \\
\hline 89.50 & $356 \pm 0.4$ & $73 \pm 2$ & $48,885 \pm 1,451$ & $84 \pm 1$ & $0.6063 \pm 0.0011$ & $88,126 \pm 311$ & $88,056 \pm 311$ & $108 \pm 2$ \\
\hline 95.75 & $326 \pm 0.3$ & $42 \pm 2$ & $77,356 \pm 2,847$ & $85 \pm 1$ & $0.6083 \pm 0.0011$ & $88,437 \pm 303$ & $88,371 \pm 303$ & $109 \pm 2$ \\
\hline 99.50 & $430 \pm 0.4$ & $10 \pm 1$ & $420,386 \pm 51,620$ & $85 \pm 1$ & $0.6096 \pm 0.0010$ & $88,679 \pm 286$ & $88,614 \pm 286$ & $109 \pm 2$ \\
\hline 104.00 & $386 \pm 0.3$ & $587 \pm 12$ & $6,623 \pm 135$ & $87 \pm 1$ & $0.6109 \pm 0.0009$ & $88,722 \pm 262$ & $88,615 \pm 262$ & $112 \pm 2$ \\
\hline 110.00 & $363 \pm 0,3$ & $93 \pm 2$ & $39,258 \pm 1,006$ & $86 \pm 1$ & $0.6111 \pm 0.0008$ & $88,899 \pm 267$ & $88,825 \pm 267$ & $111 \pm 2$ \\
\hline 117.00 & $426 \pm 0.4$ & $29 \pm 2$ & $146,933 \pm 7,574$ & $90 \pm 2$ & $0.6144 \pm 0.0008$ & $89,064 \pm 277$ & $88,995 \pm 277$ & $116 \pm 2$ \\
\hline 123.75 & $414 \pm 0.4$ & $48 \pm 2$ & $86,637 \pm 4,324$ & $87 \pm 1$ & $0.6138 \pm 0.0009$ & $89,403 \pm 268$ & $89,333 \pm 268$ & $112 \pm 2$ \\
\hline 155.75 & $436 \pm 0.6$ & $106 \pm 3$ & $42,484 \pm 1,049$ & $103 \pm 2$ & $0.6290 \pm 0.0012$ & $90,455 \pm 385$ & $90,386 \pm 385$ & $134 \pm 3$ \\
\hline 180.75 & $412 \pm 0.4$ & $56 \pm 2$ & $76,195 \pm 2,723$ & $93 \pm 1$ & $0.6235 \pm 0.0011$ & $90,742 \pm 322$ & $90,672 \pm 322$ & $120 \pm 2$ \\
\hline 204.50 & $425 \pm 0.5$ & $77 \pm 2$ & $56,793 \pm 1,443$ & $93 \pm 2$ & $0.6246 \pm 0.0012$ & $90,922 \pm 363$ & $90,856 \pm 363$ & $120 \pm 2$ \\
\hline \multicolumn{9}{|l|}{ GAS27 } \\
\hline 152.75 & $255 \pm 0.3$ & $49 \pm 2$ & $60,104 \pm 2,379$ & $135 \pm 1$ & $0.7053 \pm 0.0012$ & $103,176 \pm 364$ & $103,105 \pm 364$ & $180 \pm 2$ \\
\hline 162.75 & $583 \pm 0.7$ & $25 \pm 2$ & $269,724 \pm 18,755$ & $132 \pm 2$ & $0.7054 \pm 0.0010$ & $103,586 \pm 342$ & $103,518 \pm 342$ & $177 \pm 2$ \\
\hline 169.00 & $344 \pm 0.4$ & $9 \pm 1$ & $420,035 \pm 46,871$ & $128 \pm 2$ & $0.7025 \pm 0.0010$ & $103,655 \pm 345$ & $103,587 \pm 345$ & $171 \pm 2$ \\
\hline 174.50 & $346 \pm 0.3$ & $13 \pm 2$ & $300,677 \pm 35,248$ & $123 \pm 1$ & $0.7004 \pm 0.0009$ & $103,941 \pm 329$ & $103,873 \pm 329$ & $165 \pm 2$ \\
\hline 181.50 & $351 \pm 0.4$ & $19 \pm 2$ & $209,908 \pm 16,984$ & $122 \pm 2$ & $0.7002 \pm 0.0016$ & $104,044 \pm 498$ & $103,980 \pm 498$ & $164 \pm 2$ \\
\hline 184.50 & $359 \pm 0.4$ & $66 \pm 2$ & $62,558 \pm 1,685$ & $120 \pm 2$ & $0.6993 \pm 0.0010$ & $104,130 \pm 370$ & $104,058 \pm 370$ & $162 \pm 2$ \\
\hline 188.25 & $377 \pm 0.3$ & $20 \pm 1$ & $219,403 \pm 15,795$ & $119 \pm 1$ & $0.7003 \pm 0.0010$ & $104,542 \pm 339$ & $104,473 \pm 339$ & $160 \pm 2$ \\
\hline 196.00 & $328 \pm 0.4$ & $7 \pm 1$ & $559,710 \pm 85,363$ & $116 \pm 2$ & $0.6980 \pm 0.0010$ & $104,623 \pm 361$ & $104,555 \pm 361$ & $155 \pm 2$ \\
\hline 209.75 & $327 \pm 0.3$ & $79 \pm 2$ & $47,623 \pm 1,481$ & $109 \pm 2$ & $0.6938 \pm 0.0014$ & $104,788 \pm 437$ & $104,715 \pm 437$ & $146 \pm 2$ \\
\hline \multicolumn{9}{|l|}{ GAS29 } \\
\hline 51.25 & $330 \pm 0.3$ & $291 \pm 6$ & $13,228 \pm 276$ & $129 \pm 1$ & $0.7077 \pm 0.0010$ & $104,658 \pm 349$ & $104,573 \pm 350$ & $174 \pm 2$ \\
\hline 56.25 & $283 \pm 1$ & $32 \pm 1$ & $104,411 \pm 571$ & $125 \pm 1$ & $0.7053 \pm 0.0010$ & $104,810 \pm 332$ & $104,740 \pm 332$ & $168 \pm 2$ \\
\hline 62.50 & $332 \pm 0.3$ & $16 \pm 1$ & $236,755 \pm 21,567$ & $129 \pm 1$ & $0.7100 \pm 0.0011$ & $105,271 \pm 354$ & $105,207 \pm 354$ & $174 \pm 2$ \\
\hline 78.75 & $346 \pm 0.4$ & $147 \pm 3$ & $27,358 \pm 617$ & $122 \pm 2$ & $0.7064 \pm 0.0010$ & $105,549 \pm 368$ & $105,475 \pm 368$ & $165 \pm 2$ \\
\hline 97.50 & $348 \pm 0.4$ & $118 \pm 3$ & $34,306 \pm 847$ & $118 \pm 1$ & $0.7053 \pm 0.0014$ & $106,026 \pm 416$ & $105,954 \pm 416$ & $159 \pm 2$ \\
\hline 113.75 & $273 \pm 0.3$ & $133 \pm 3$ & $23,937 \pm 540$ & $117 \pm 1$ & $0.7073 \pm 0.0012$ & $106,717 \pm 380$ & $106,642 \pm 380$ & $158 \pm 2$ \\
\hline
\end{tabular}


Table S1: MC-ICP-MS U-Th dating results of samples analysed in this study.

$\mathbf{m m ~ d f t ~}=$ distance from top in $\mathbf{m m}$.

All uncertainties are $2 \sigma$.

$* \delta^{234} \mathrm{U}=\left(\left[{ }^{234} \mathrm{U} /{ }^{238} \mathrm{U}\right]\right.$ activity-1)x 1000 .

$\dagger\left[{ }^{230} \mathrm{Th} /{ }^{238} \mathrm{U}\right]_{\text {activity }}=1-\mathrm{e}^{-\lambda 230 \mathrm{~T}}+\left(\delta^{234} \mathrm{U}_{\text {measured }} / 1000\right)\left[\lambda_{230} /\left(\lambda_{230}-\lambda_{234}\right)\right]\left(1-\mathrm{e}^{-(\lambda 230-\lambda 234) \mathrm{T}}\right)$, where $\mathrm{T}$ is age in years. $\lambda_{230}=9.1705 \times 10^{-6} \mathrm{a}^{-1}(\mathrm{Cheng}$ et al., 2013),

$\lambda_{234}=2.8221 \times 10^{-6} \mathrm{a}^{-1}$ (Cheng et al., 2013), $\lambda^{238}=1.551 \times 10^{-1} \mathrm{a}^{-1}$ (Jaffey et al., 1971).

$\S$ Years before measurement and without detrital Th correction.

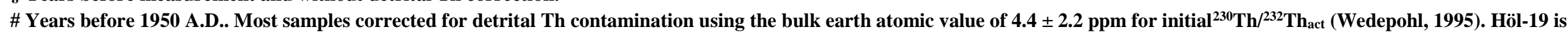

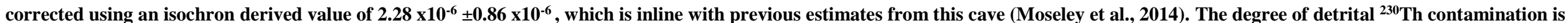

indicated by the measured $\left({ }^{230} \mathrm{Th} /{ }^{232} \mathrm{Th}\right)$ activity ratio. 
Age (years before 1950 A.D.)

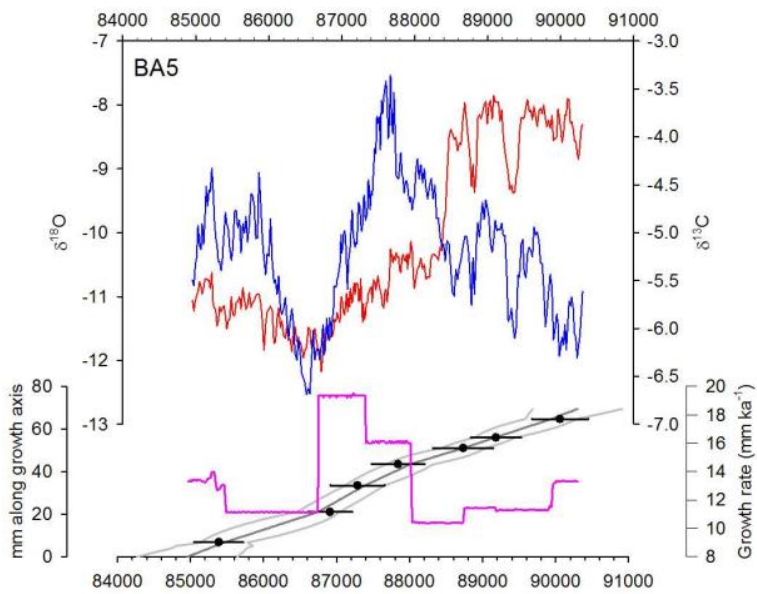

Age (years before 1950 A.D.)

Age (years before 1950 A.D.)

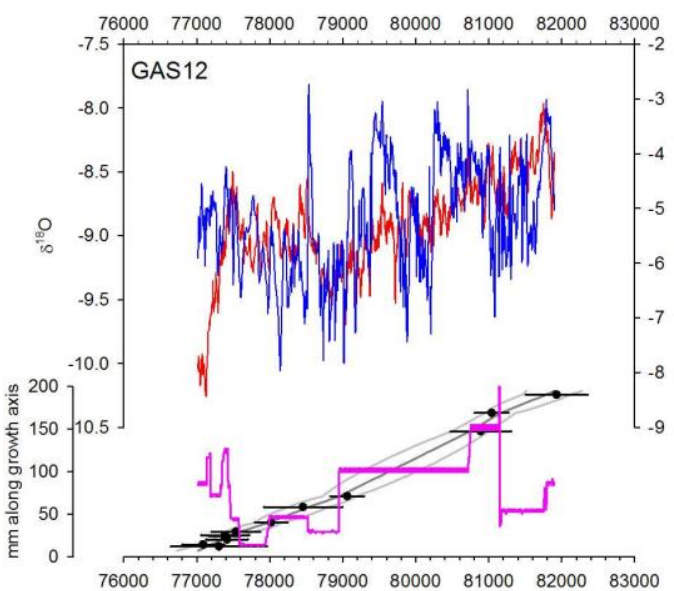

Age (years before 1950 A.D.

Age (years before 1950 A.D.)

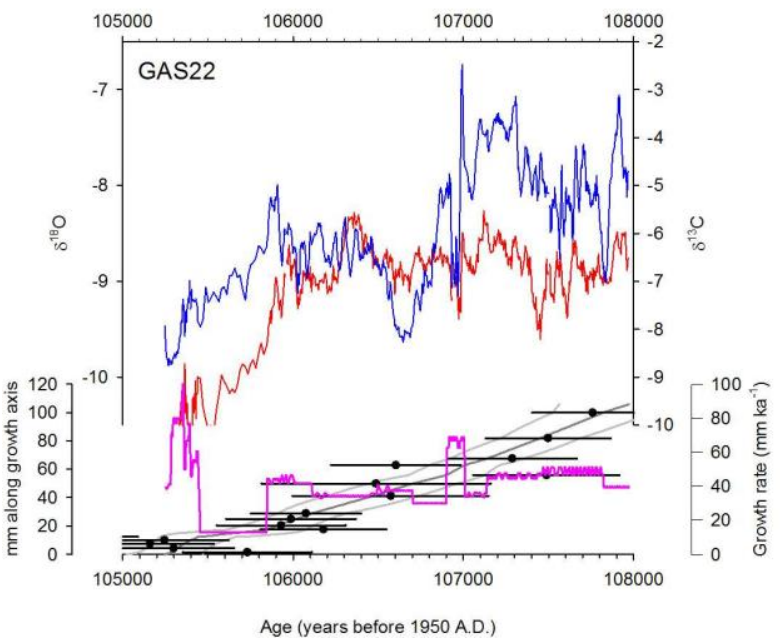

Age (years before 1950 A.D.)

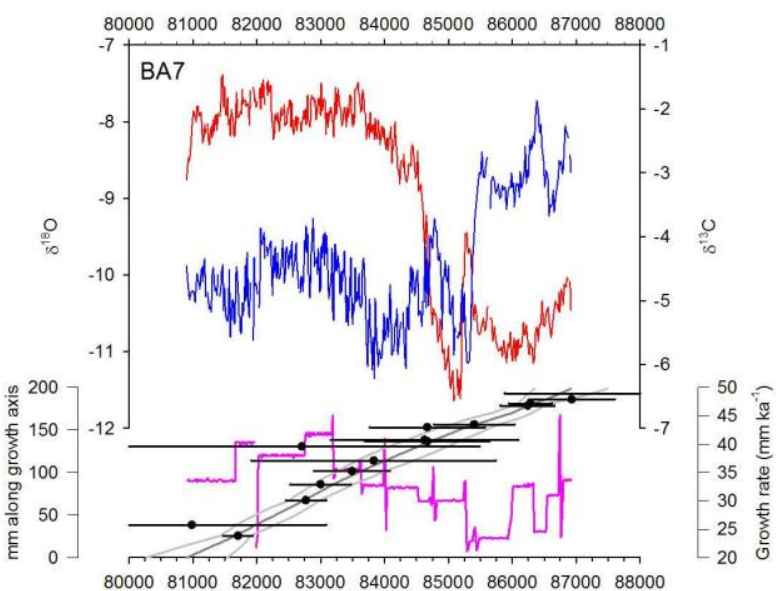

Age (years before 1950 A.D.)

Age (years before 1950 A.D.)

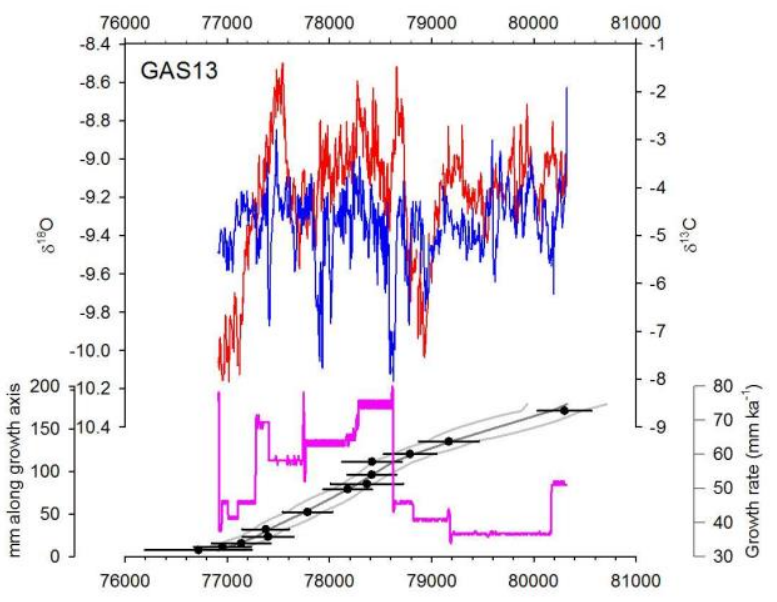

Age (years before 1950 A.D.)

Age (years before 1950 A.D.)

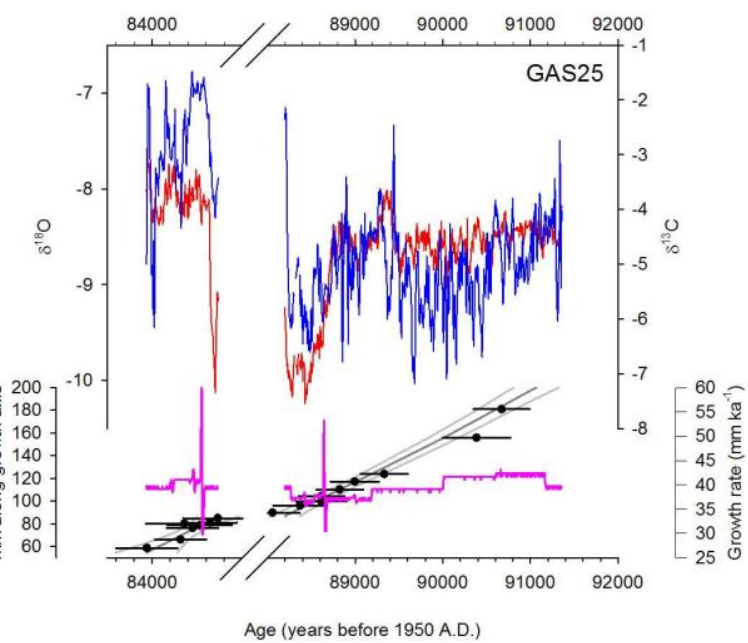




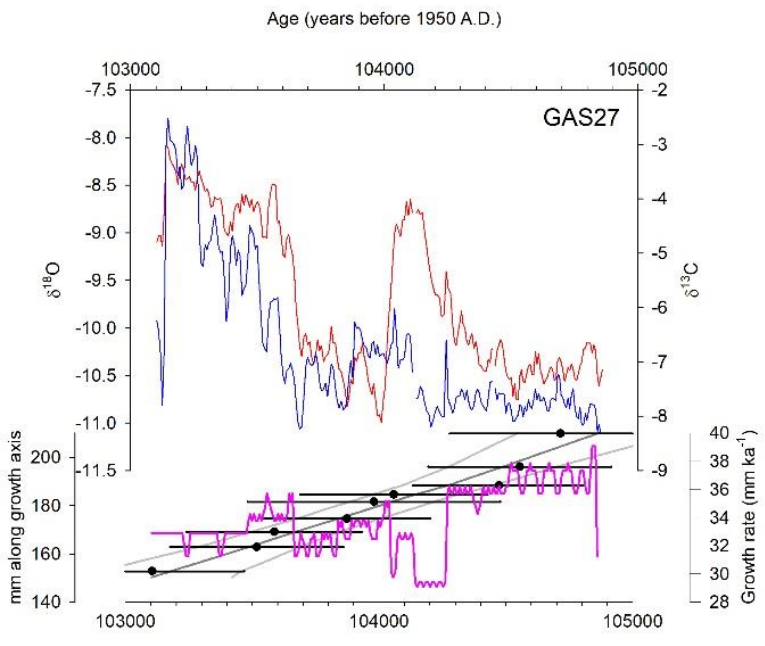

Age (years before 1950 A.D.)

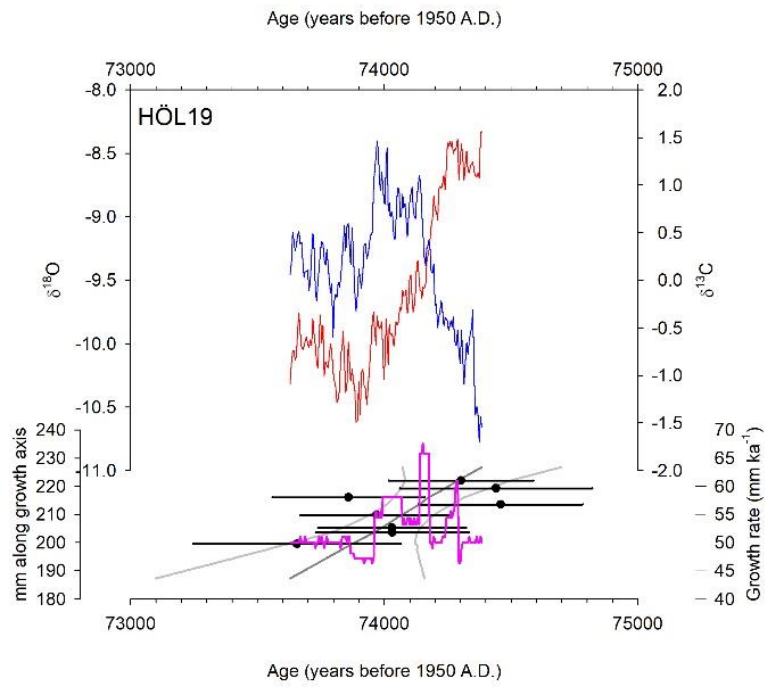

Age (years before 1950 A.D.)

Age (years before 1950 A.D.)

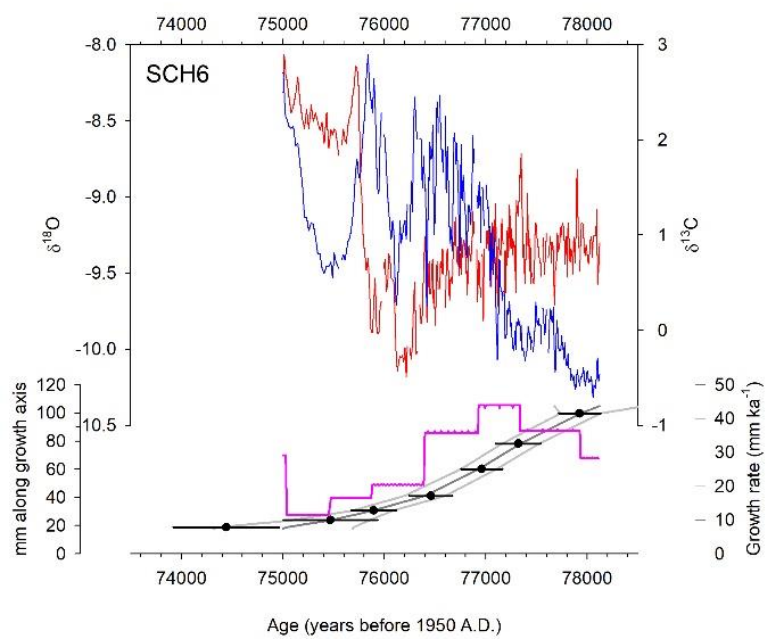

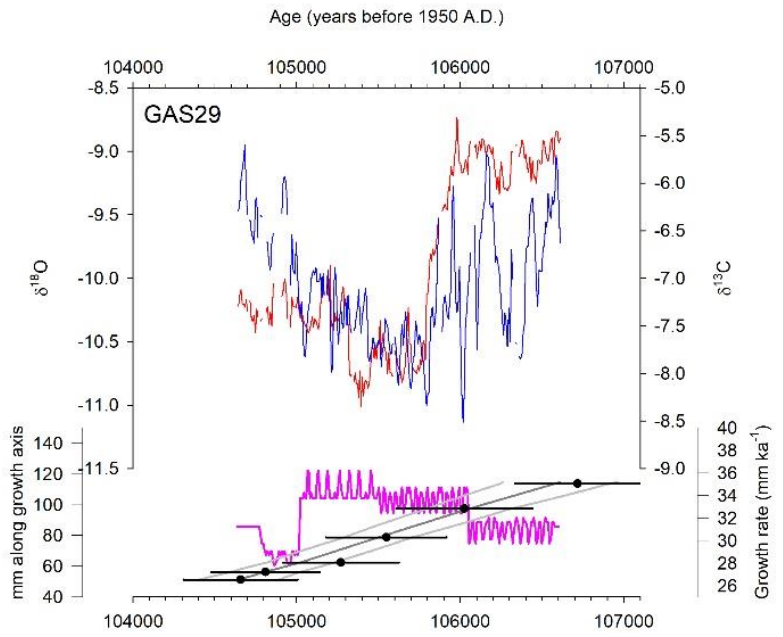

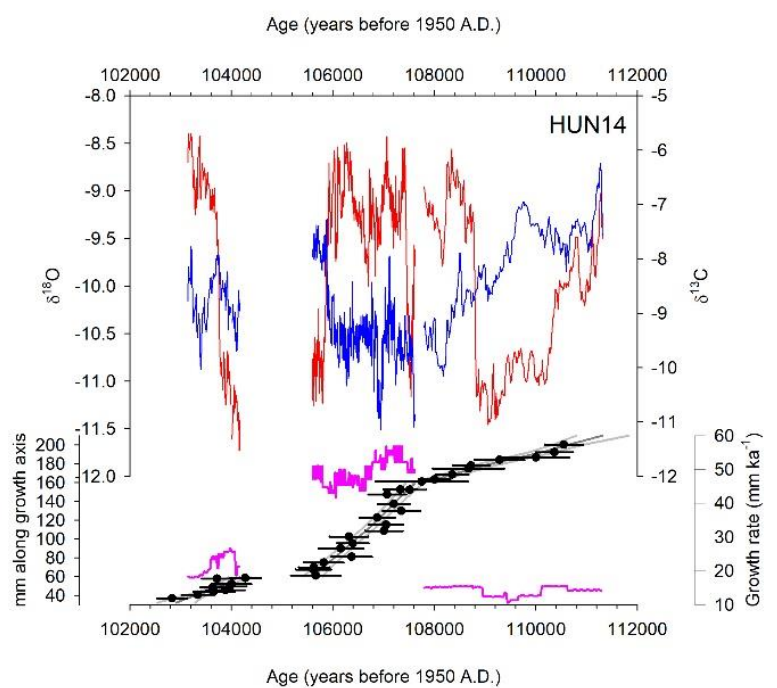

Figure S3: Age modelling results for samples analysed in this study. Upper graphs show $\delta^{18} O$ (red) and $\delta^{13} \mathrm{C}$ (blue) plotted on the Oxcal age model. Lower graphs show the U-Th ages with 2 sigma uncertainty used in the Oxcal age model (black circles with horizontal lines) plotted relative to distance along the growth axis. The dark grey line marks the central age model and the light grey lines mark the 2 sigma uncertainty. Pink lines show the growth rate. 

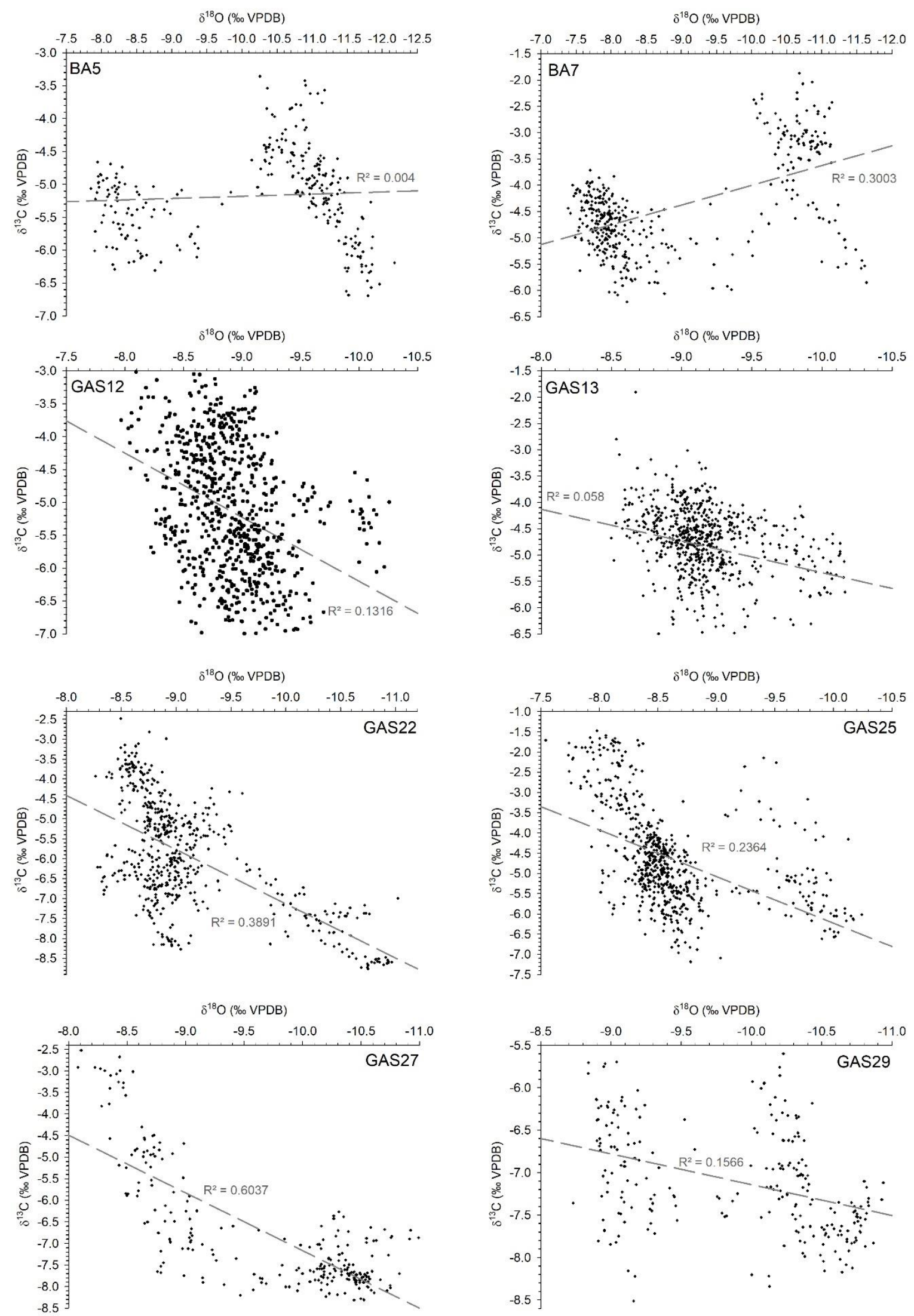

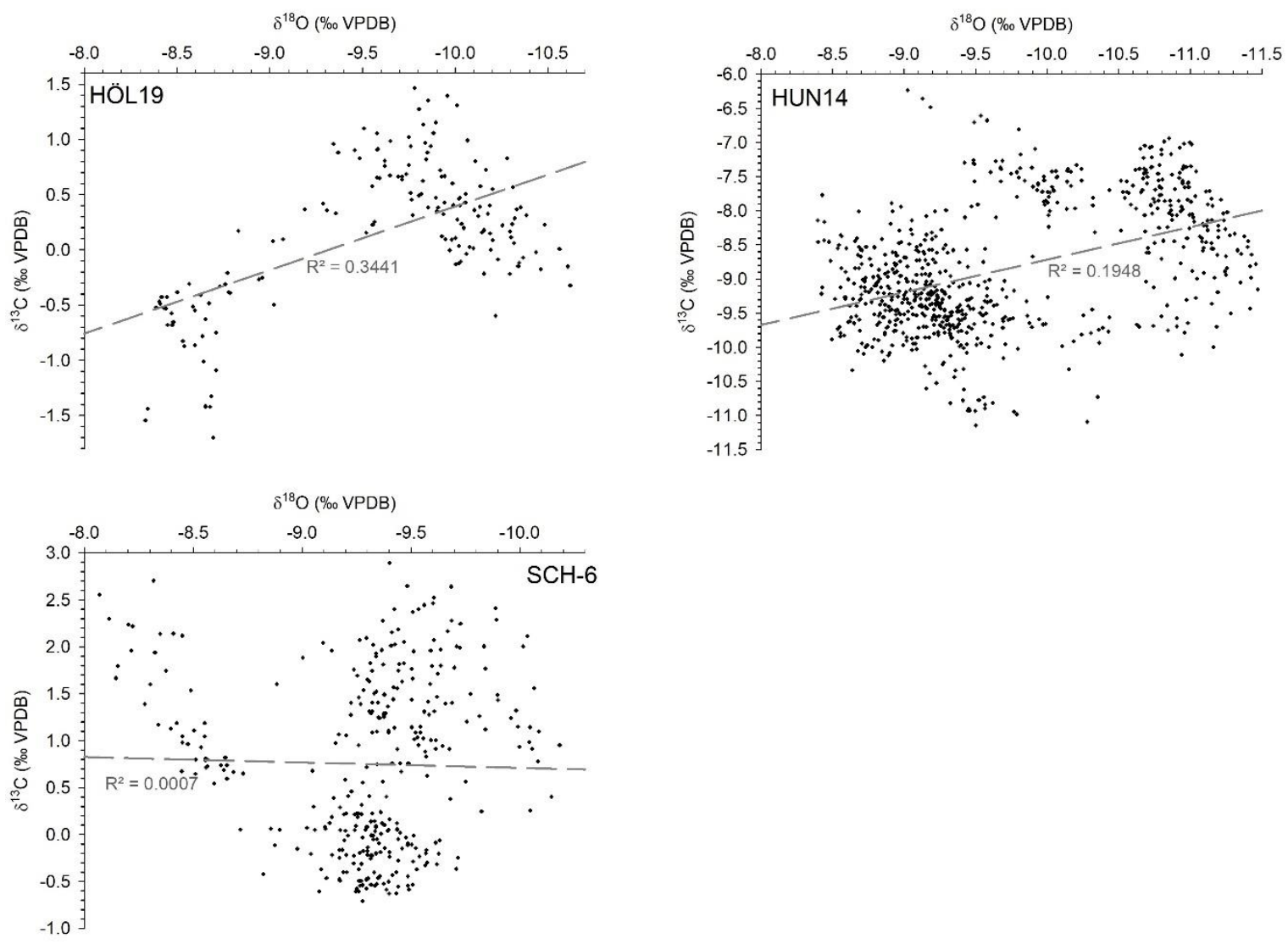

Figure S4: $\delta^{18} \mathrm{O}$ versus $\delta^{13} \mathrm{C}$ for each sample analysed in this study as well as the correlation coefficient $\left(\mathbf{R}^{2}\right)$.

\begin{tabular}{|c|c|c|c|c|c|c|c|c|c|c|c|c|}
\hline Sample & \multicolumn{2}{|l|}{$\mathrm{H} 1$} & \multicolumn{2}{|l|}{$\mathrm{H} 2$} & \multicolumn{2}{|l|}{$\mathrm{H} 3$} & \multicolumn{2}{|l|}{$\mathrm{H} 4$} & \multicolumn{2}{|l|}{$\mathrm{H} 5$} & \multicolumn{2}{|l|}{$\mathrm{H} 6$} \\
\hline & $\delta^{13} \mathrm{C}$ & $\delta^{18} \mathrm{O}$ & $\delta^{13} \mathrm{C}$ & $\delta^{18} \mathrm{O}$ & $\delta^{13} \mathrm{C}$ & $\delta^{18} \mathrm{O}$ & $\delta^{13} \mathrm{C}$ & $\delta^{18} \mathrm{O}$ & $\delta^{13} \mathrm{C}$ & $\delta^{18} \mathrm{O}$ & $\delta^{13} C$ & $\delta^{18} \mathrm{O}$ \\
\hline BA5 & 0.2 & 0.2 & 0.3 & 0.4 & & & & & & & & \\
\hline BA7 & 0.5 & 0.3 & 0.4 & 0.3 & 0.3 & 0.3 & 0.4 & 0.4 & & & & \\
\hline $\mathrm{SCH} 6$ & 0.9 & 0.4 & 0.3 & 0.7 & 1.0 & 0.6 & 0.4 & 0.2 & 0.5 & 0.3 & 0.3 & 0.3 \\
\hline HÖL19 & 0.4 & 0.3 & & & & & & & & & & \\
\hline HUN14 & 0.9 & 0.2 & 0.6 & 0.4 & 0.5 & 0.2 & 0.3 & 0.3 & & & & \\
\hline GAS22 & 2.8 & 0.4 & 1.6 & 0.5 & 3.2 & 0.3 & 1.6 & 0.3 & 2.3 & 0.4 & & \\
\hline GAS25 & 2.0 & 0.4 & 1.9 & 0.2 & 1.3 & 0.6 & 0.6 & 0.2 & 0.6 & 0.2 & 0.9 & 0.2 \\
\hline GAS27 & 2.6 & 0.4 & 4.6 & 0.8 & 1.1 & 0.3 & 2.3 & 0.5 & 0.3 & 0.4 & & \\
\hline GAS29 & 3.0 & 0.3 & 1.7 & 0.7 & 1.1 & 0.5 & 1.0 & 0.3 & 0.7 & 0.2 & & \\
\hline
\end{tabular}

Table S2: Results of Hendy tests for the samples analysed in this study. 


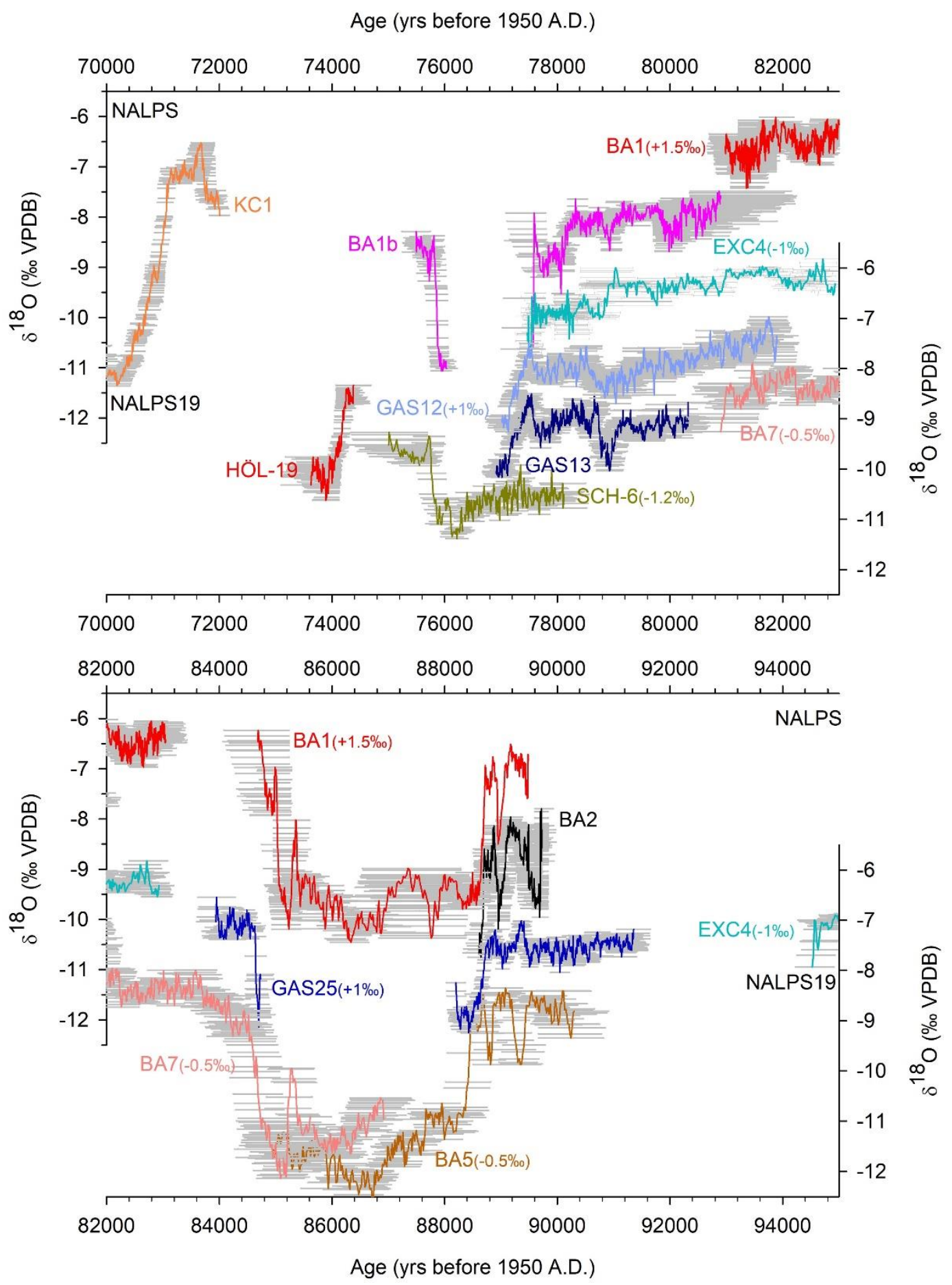


Age (yrs before 1950 A.D.)
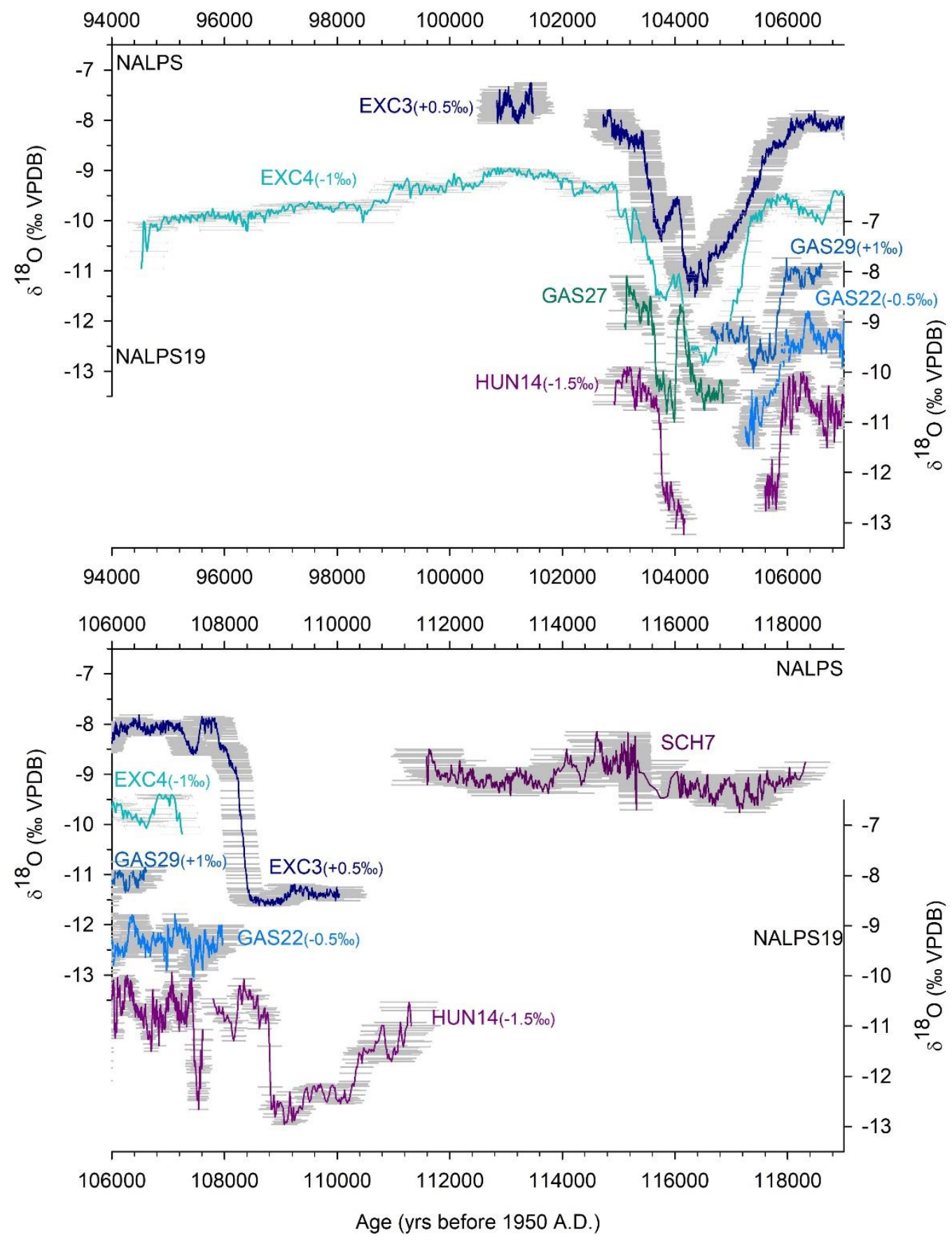

Figure S5: $\delta^{18}$ O records of NALPS19 (this study) versus NALPS11 (Boch et al., 2011) including $2 \sigma$ uncertainty (grey horizontal bars). The records are shifted for ease of viewing; the direction and amount of shift are indicated in parentheses. 
Age (yrs before 1950 A.D.)

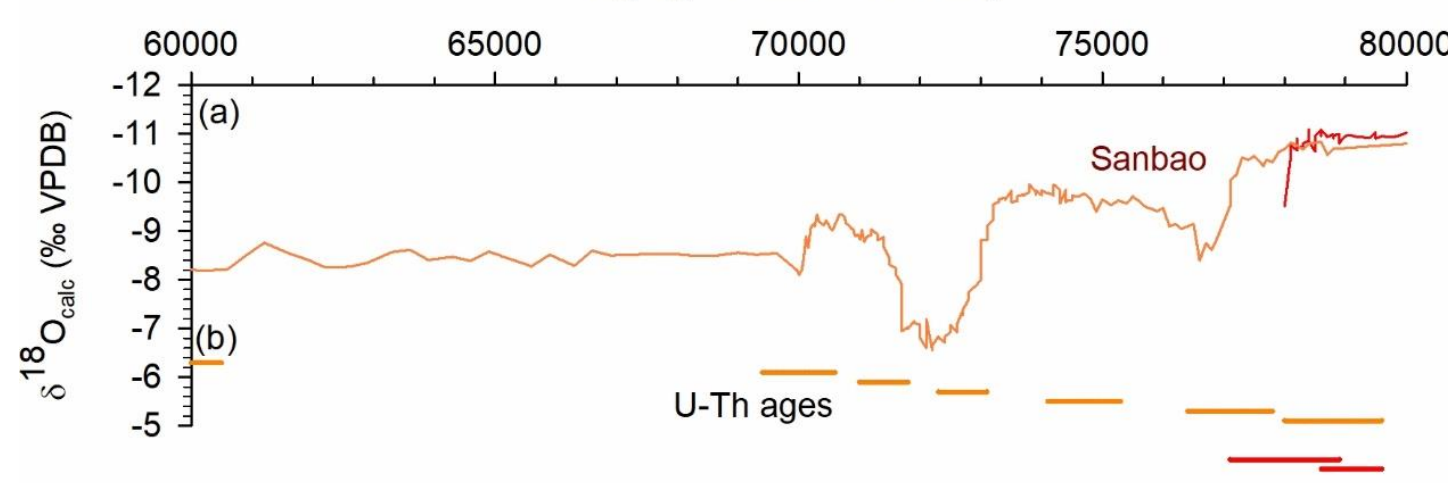

(c)

Asian monsoon composite

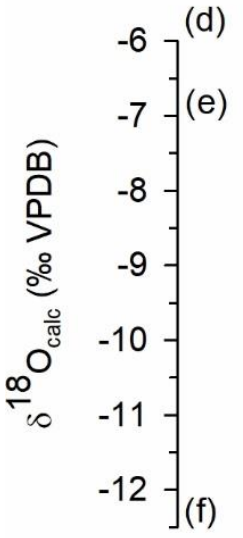

(d)

(d)

(e)

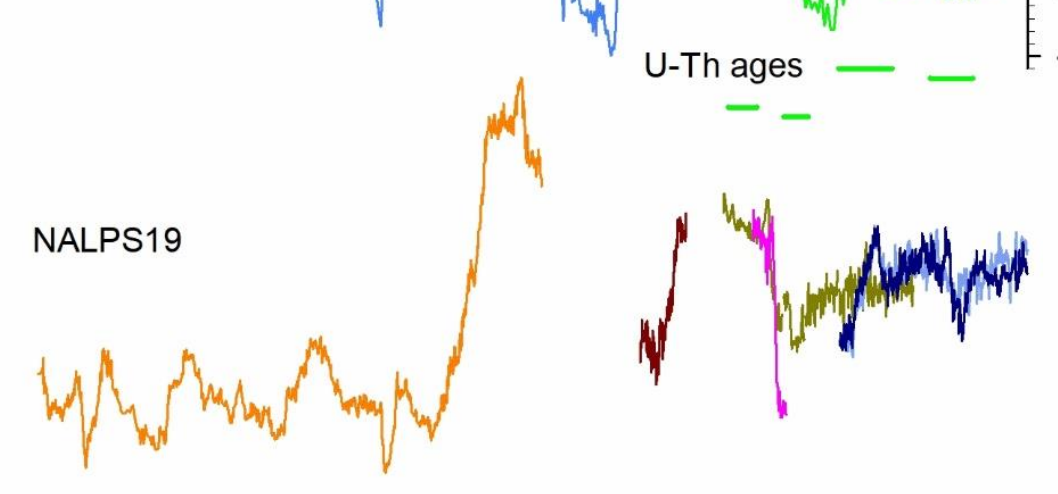

(g)
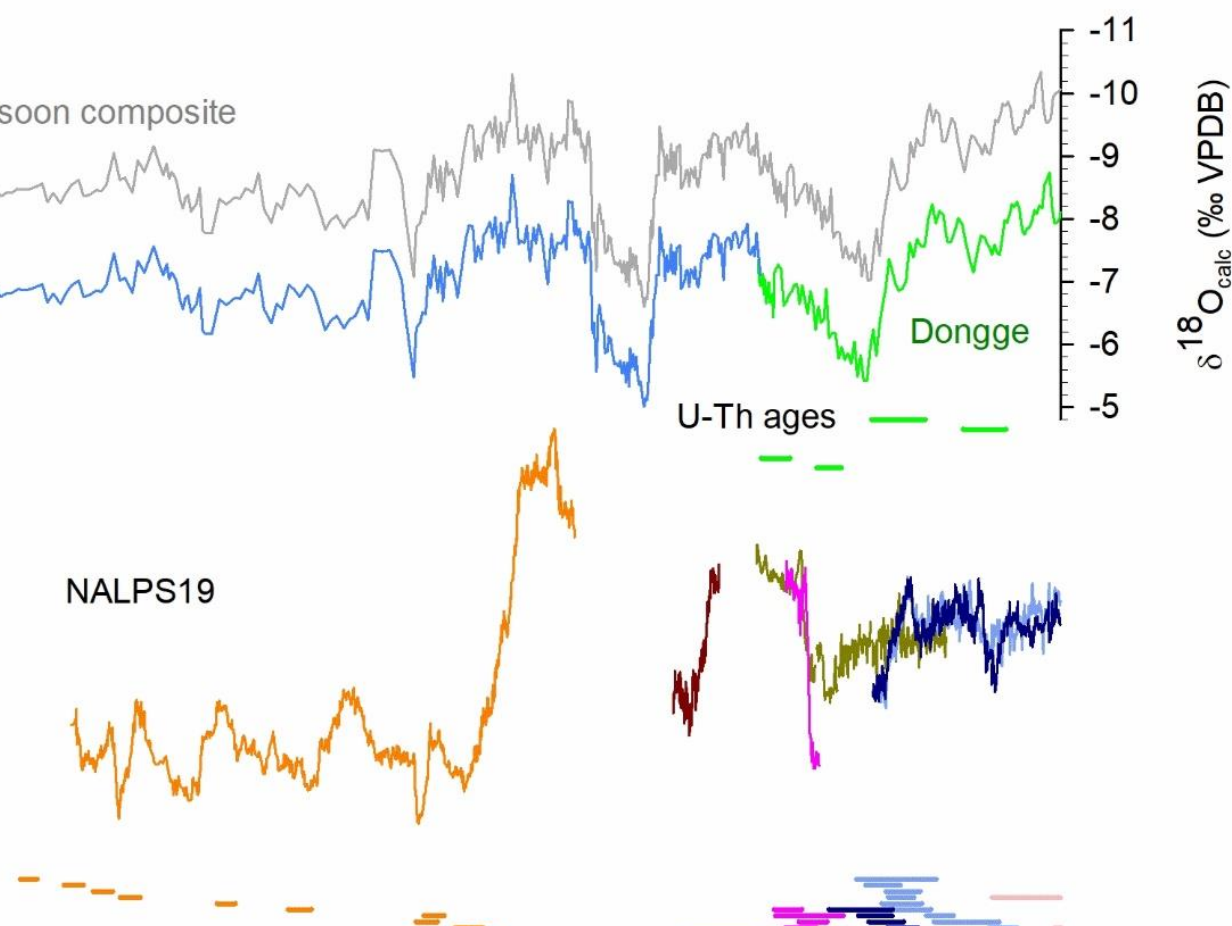

品

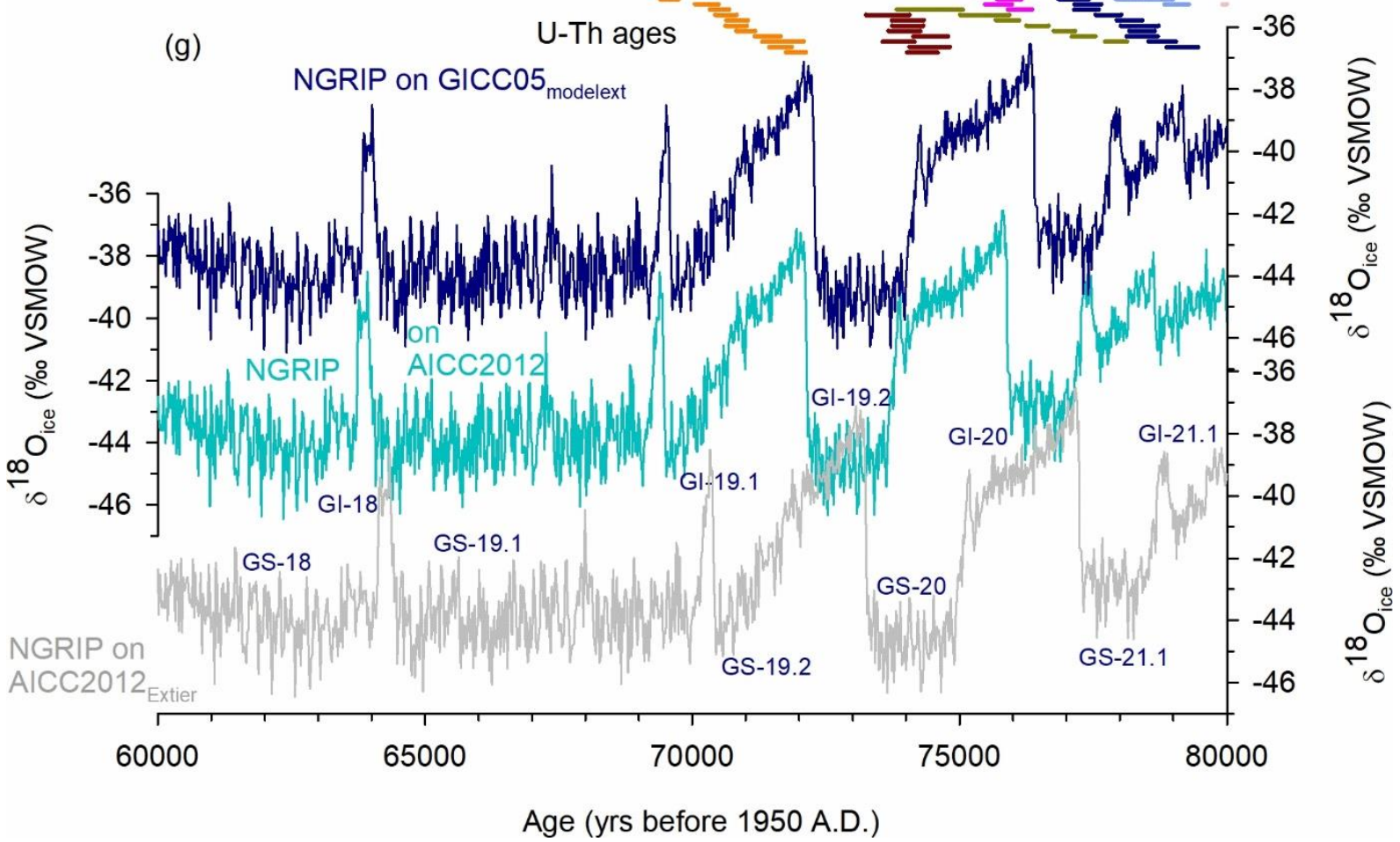




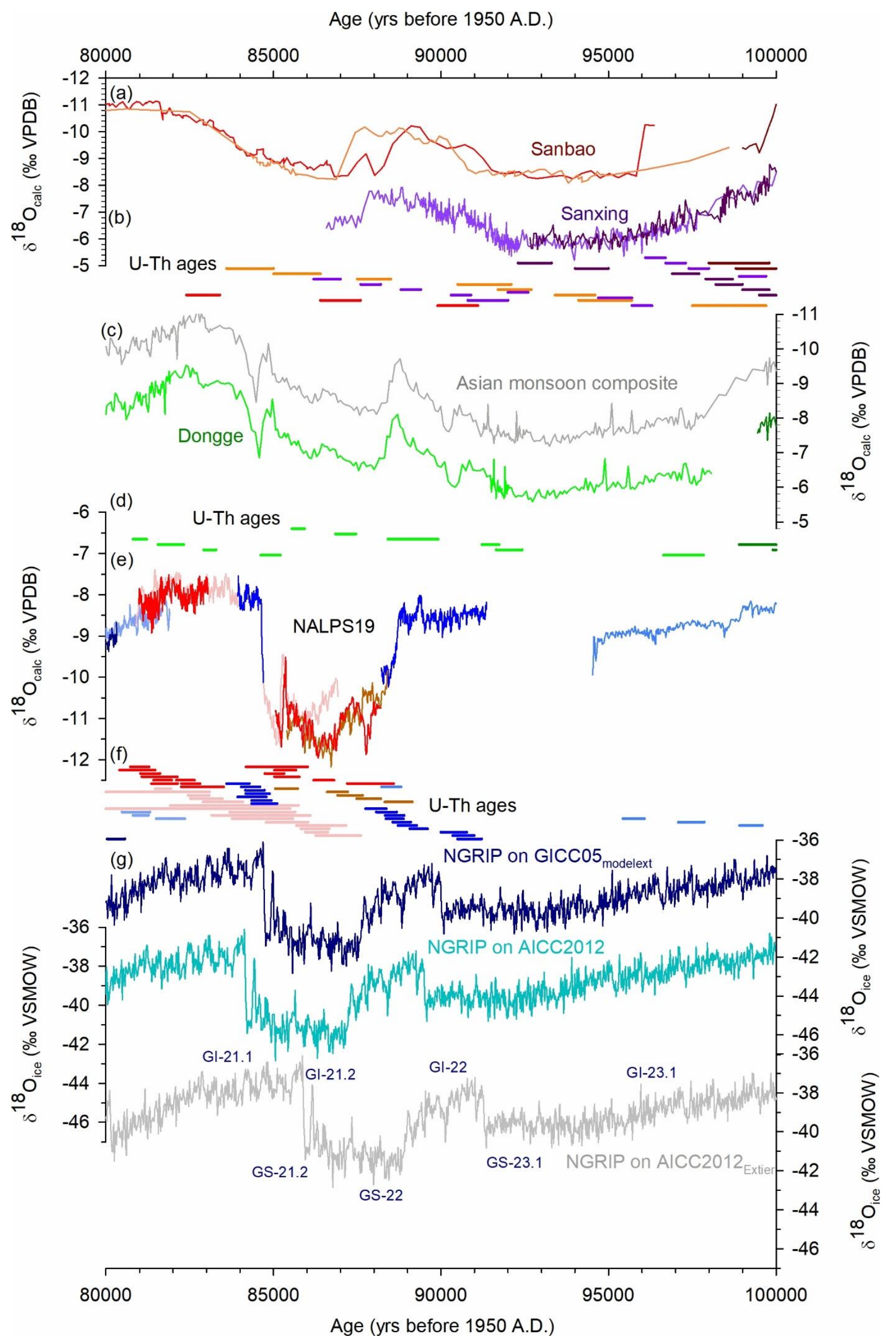



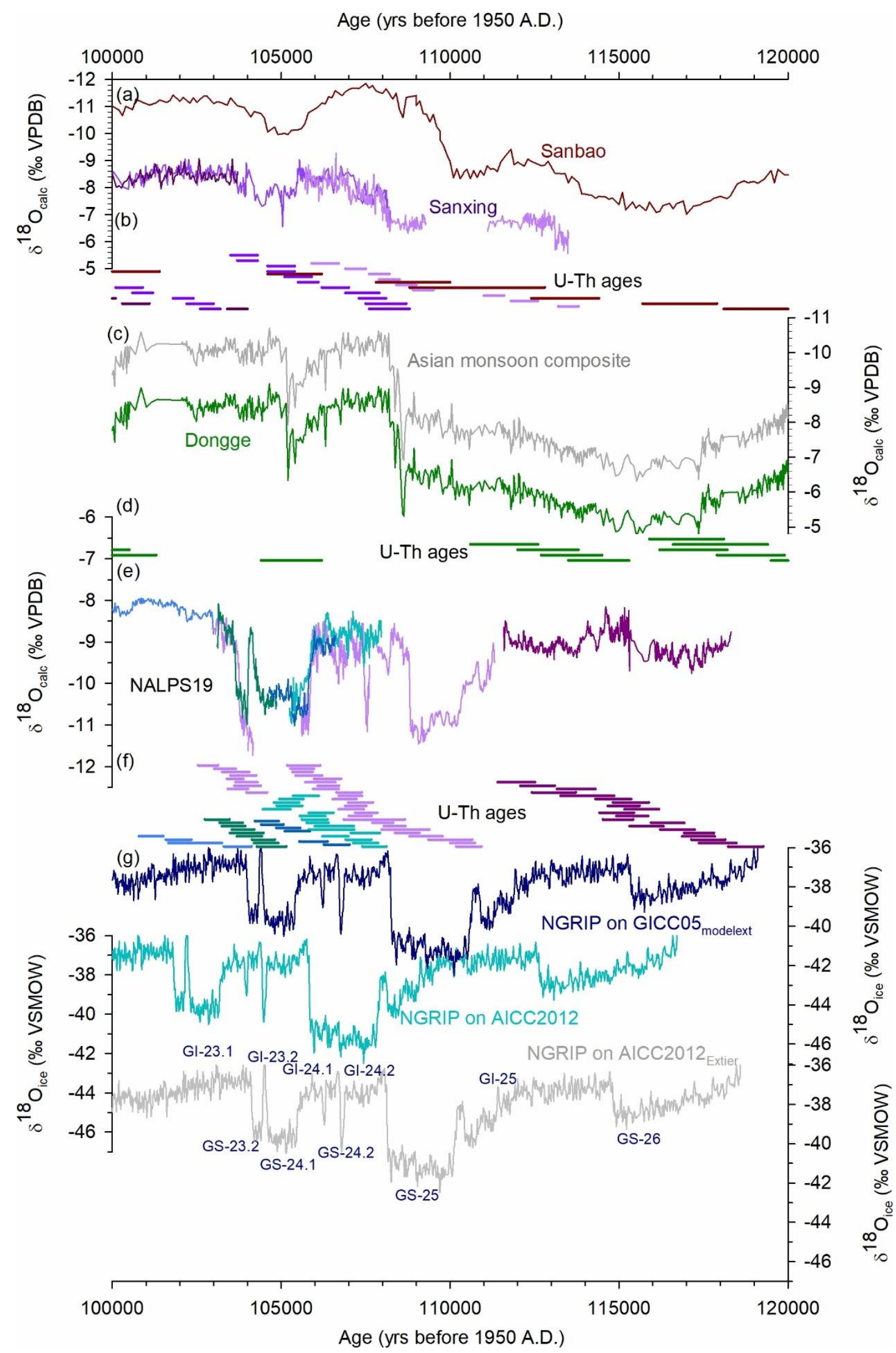

Figure S6: Fig. 3 from the main manuscript split into 20,000 year timeslices for ease of viewing. (a) Chinese speleothem $\delta^{18} O$ records from Sanbao (Wang et al., 2008) and Sanxing (Jiang et al., 2016). (b) 2 $\sigma$ range of U-Th ages for (a) colour-coded the same. (c) Asian monsoon composite record (Cheng et al., 2016) as well as the original data

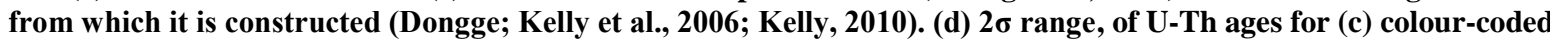
the same. (e) NALPS19 record (this study). (f) $2 \sigma$ range of $U$-Th ages for (e) colour-coded the same. (g) NGRIP

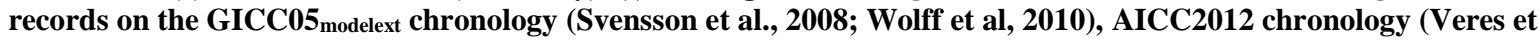
al., 2013), and AICC2012 revised according to Extier et al. (2018). 
$\mathrm{KCl}$ onset of GS-19.2

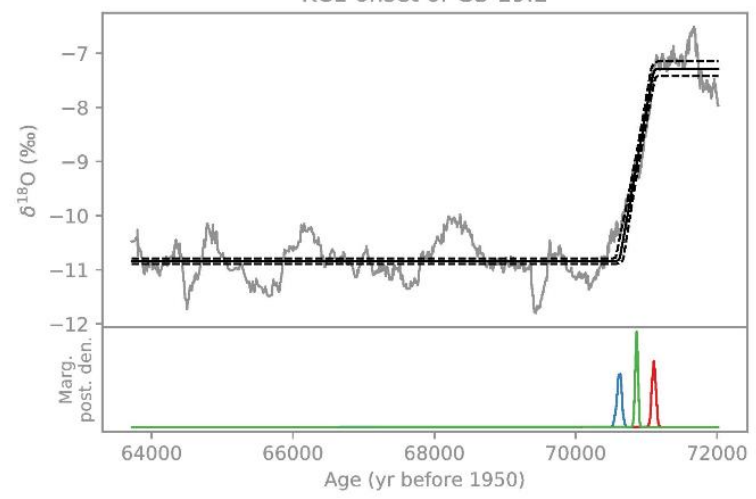

BA1b onset of GI-20

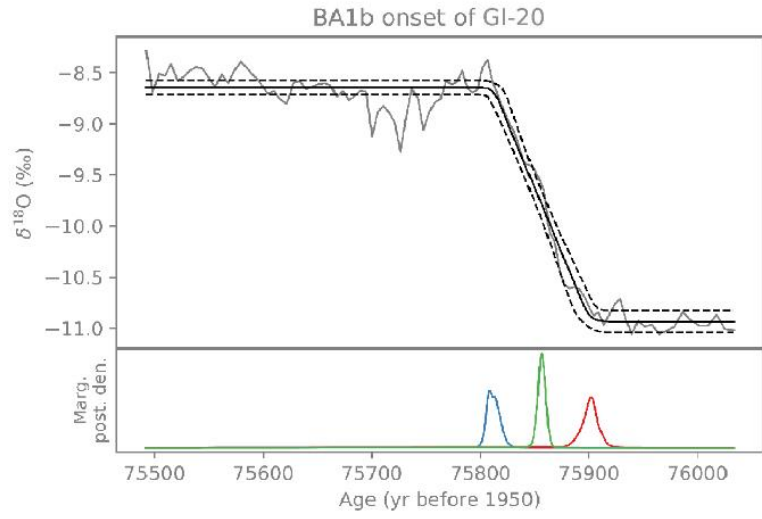

GAS-12 onset of GS-21.1

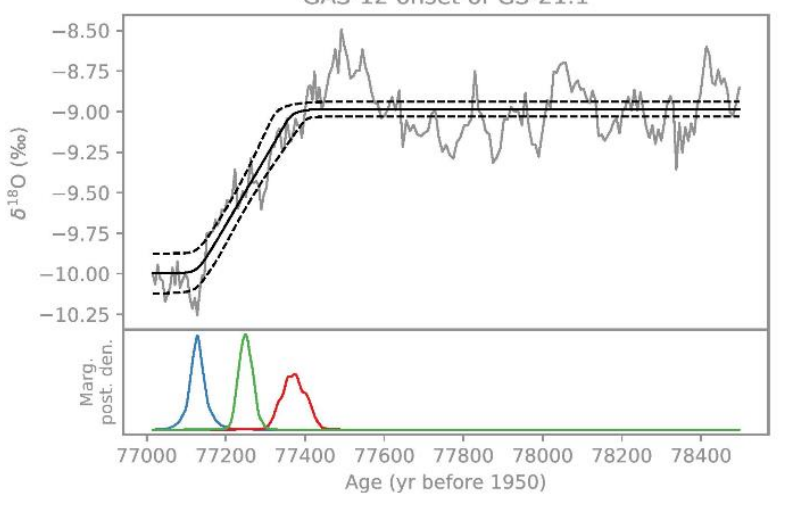

GAS25 onset of GS-22

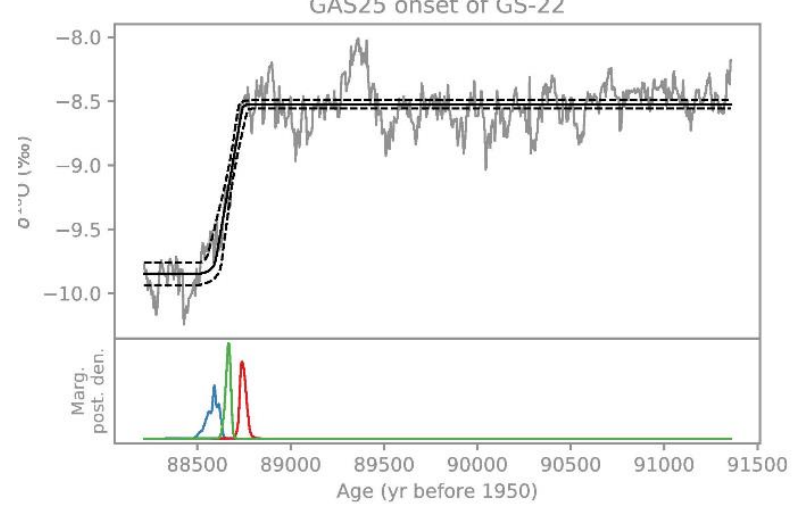

SCH-6 onset of GI-20

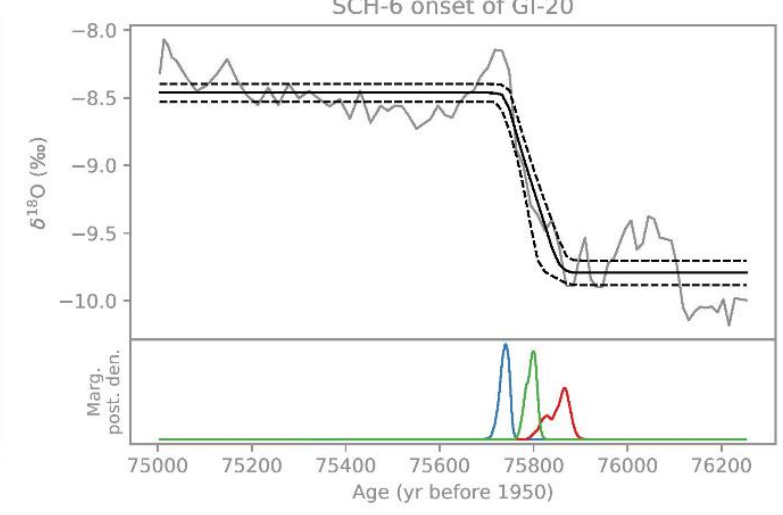

GAS-12 onset of GS-21.1

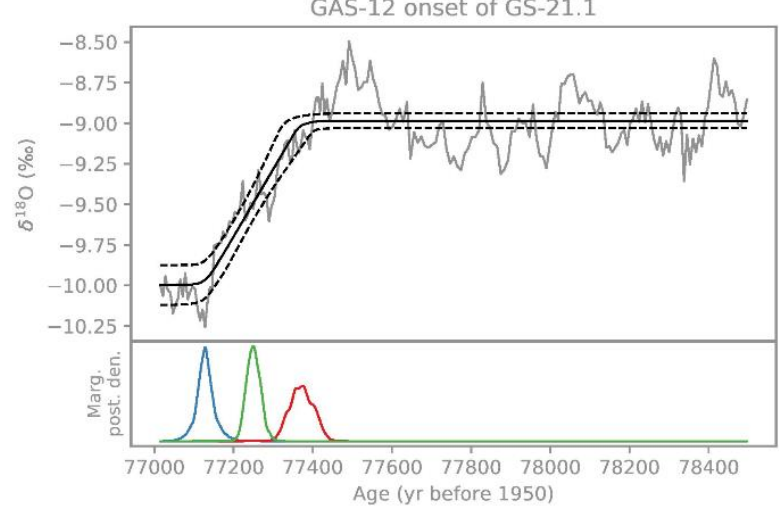

B-7-GAS-25 onset of GI-21.1

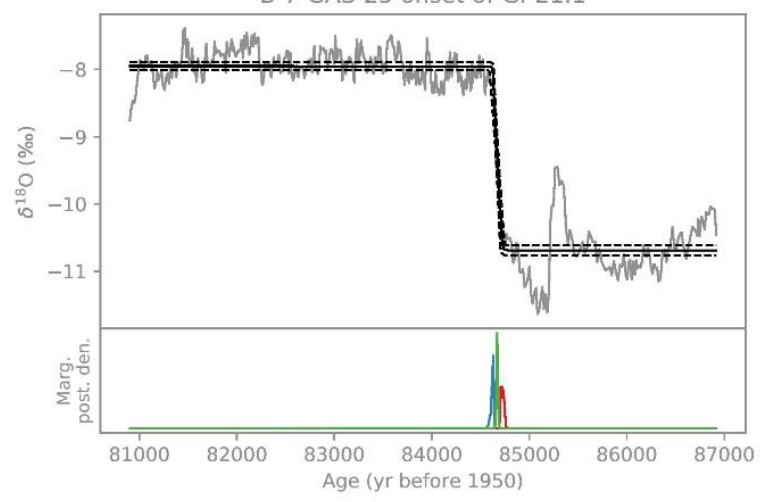

GAS-27 onset of Gl-23.1

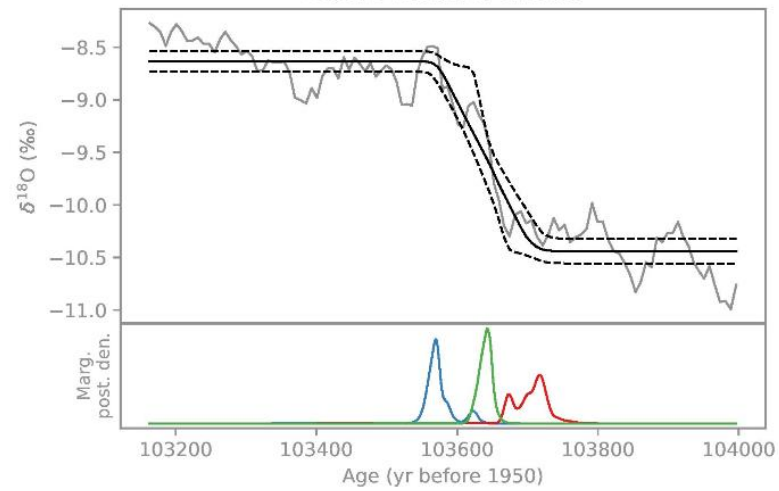


HUN-14 onset of Gl-23.1
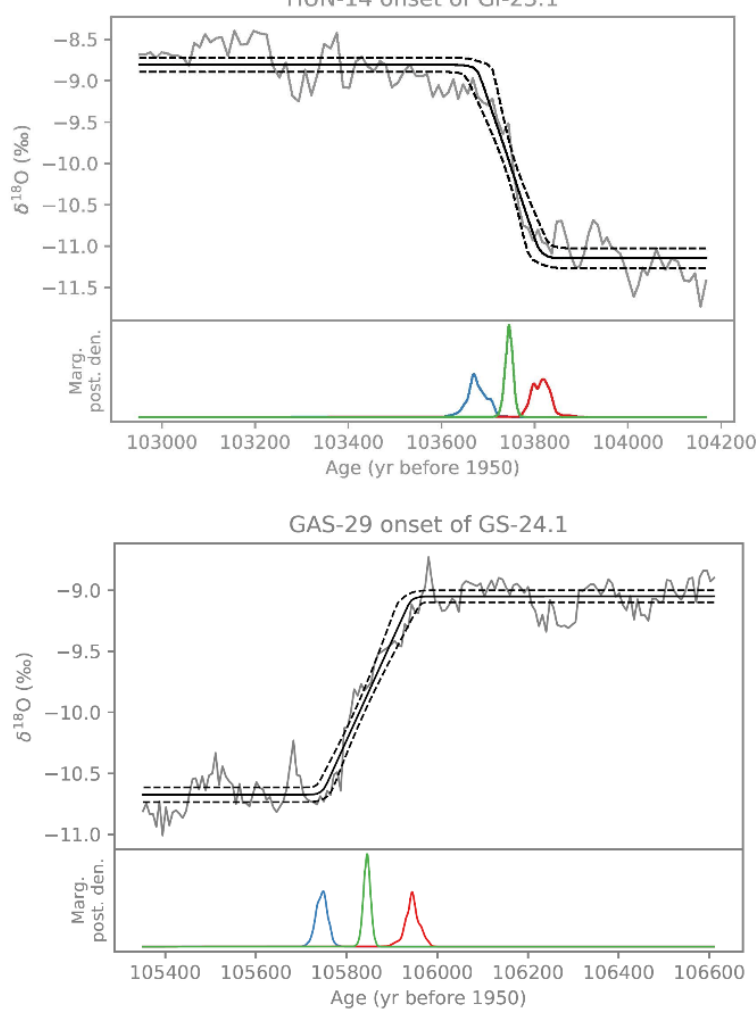

HUN-14 onset of GI-24.2

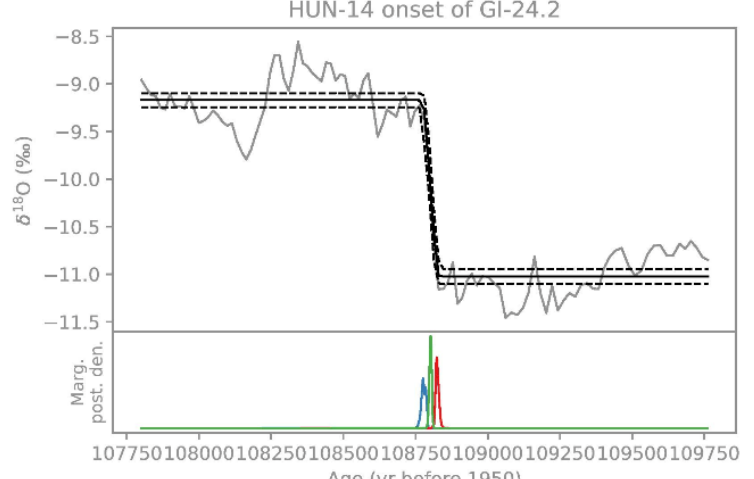

GAS-22 onset of GS-24.1

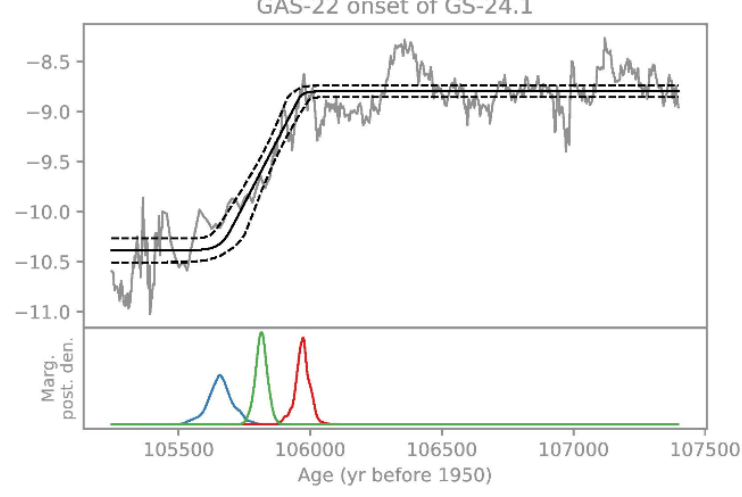

HUN-14 onset of GS-24.1

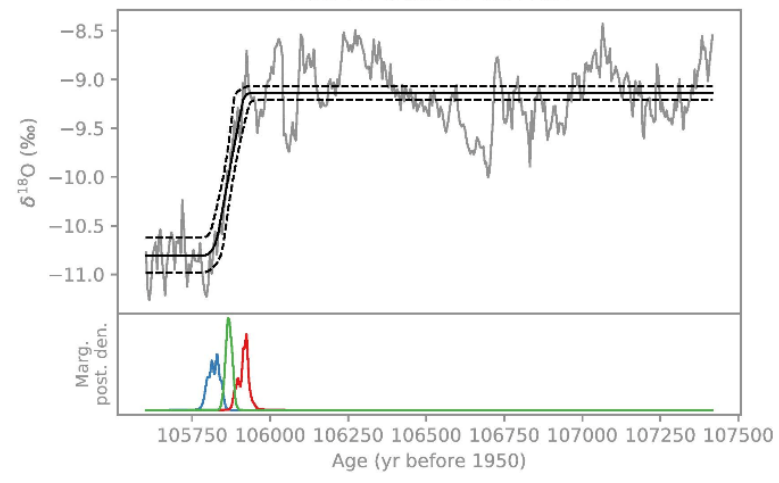

HUN-14 onset of GS-25

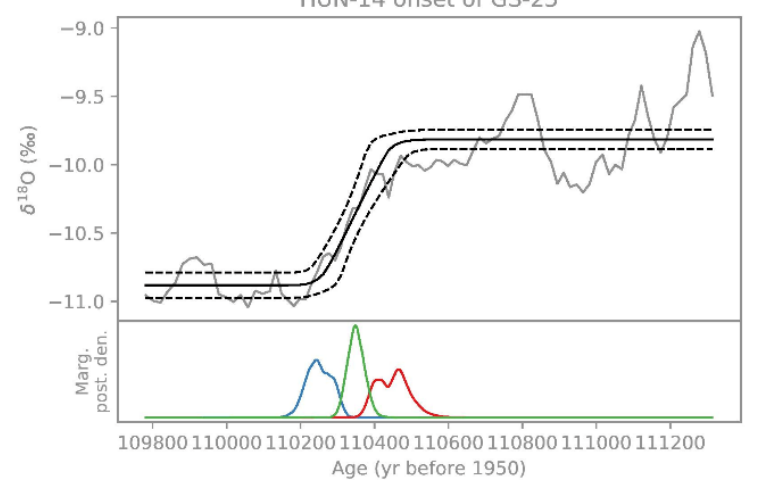

Figure S7: Fitting results of the Erhardt et al. (2019) algorithm for the NALPS19 (chronology from this study). Each of the top panels shows the fitted data together with marginal posterior median and 5 th and 95th percentile of the fitted ramp in solid and dashed lines respectively. The bottom panels show the marginal posterior densities for the onset, midpoint and endpoint of the transitions (in red, green and blue). 

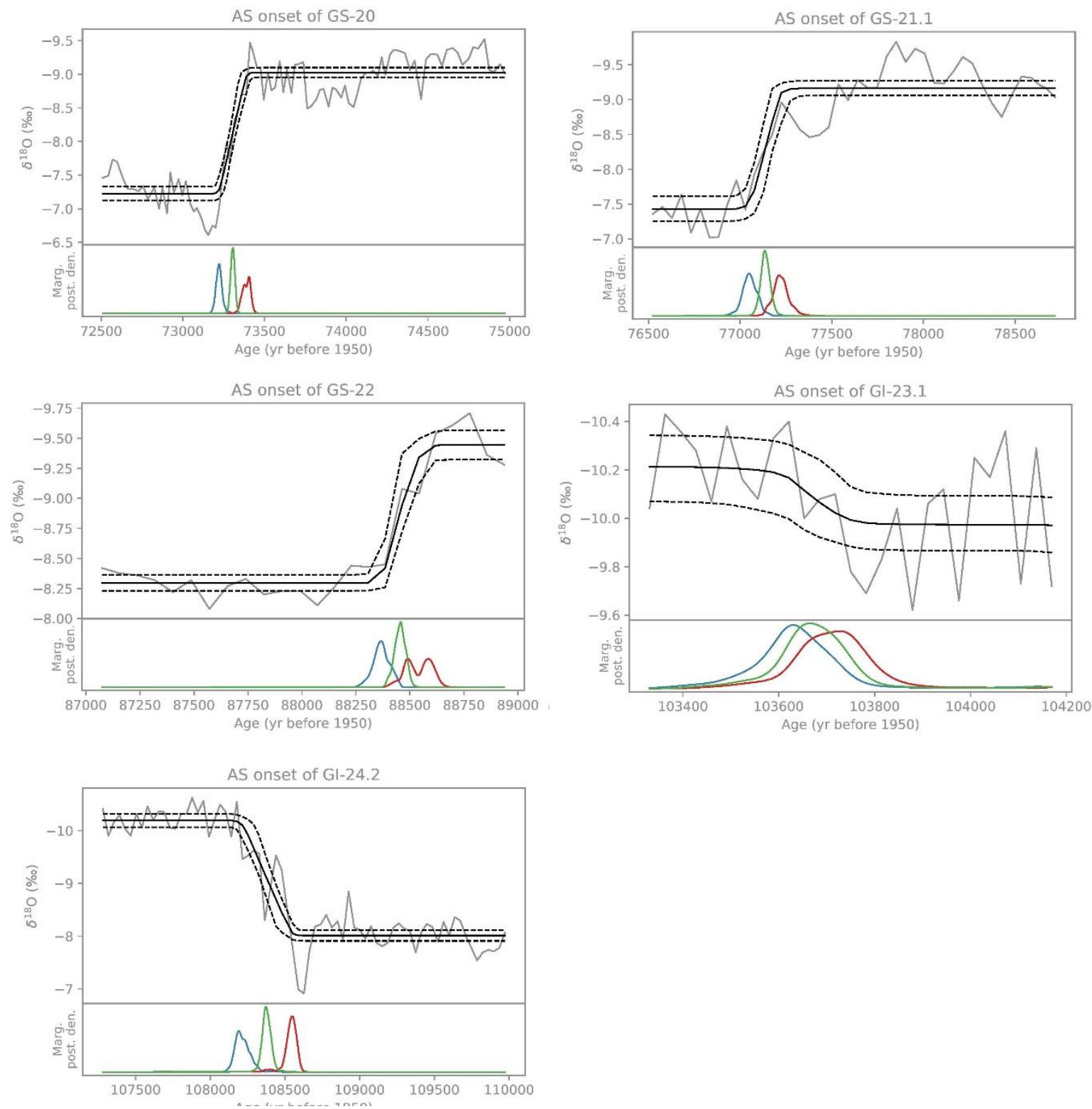

Figure S7: Fitting results of the Erhardt et al. (2019) algorithm for the Asian monsoon composite (Kelly et al., 2006; Kelly, 2010; Cheng et al., 2016). Each of the top panels shows the fitted data together with marginal posterior median and 5th and 95th percentile of the fitted ramp in solid and dashed lines respectively. The bottom panels show the marginal posterior densities for the onset, midpoint and endpoint of the transitions (in red, green and blue). 

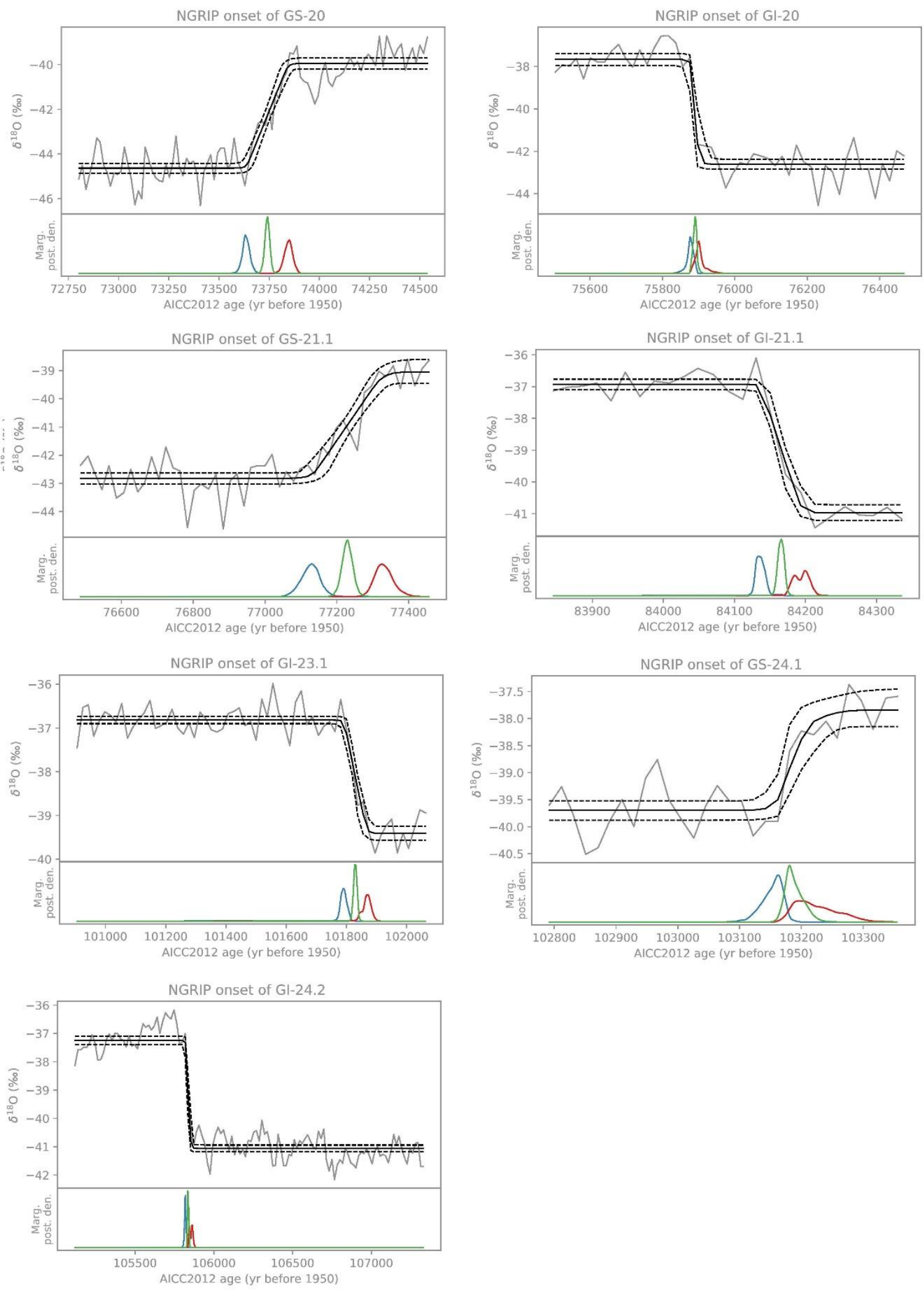

Figure S7: Fitting results of the Erhardt et al. (2019) algorithm for NGRIP on the AICC2012 chronology (Veres et al., 2013). Each of the top panels shows the fitted data together with marginal posterior median and 5th and 95th percentile of the fitted ramp in solid and dashed lines respectively. The bottom panels show the marginal posterior densities for the onset, midpoint and endpoint of the transitions (in red, green and blue). 

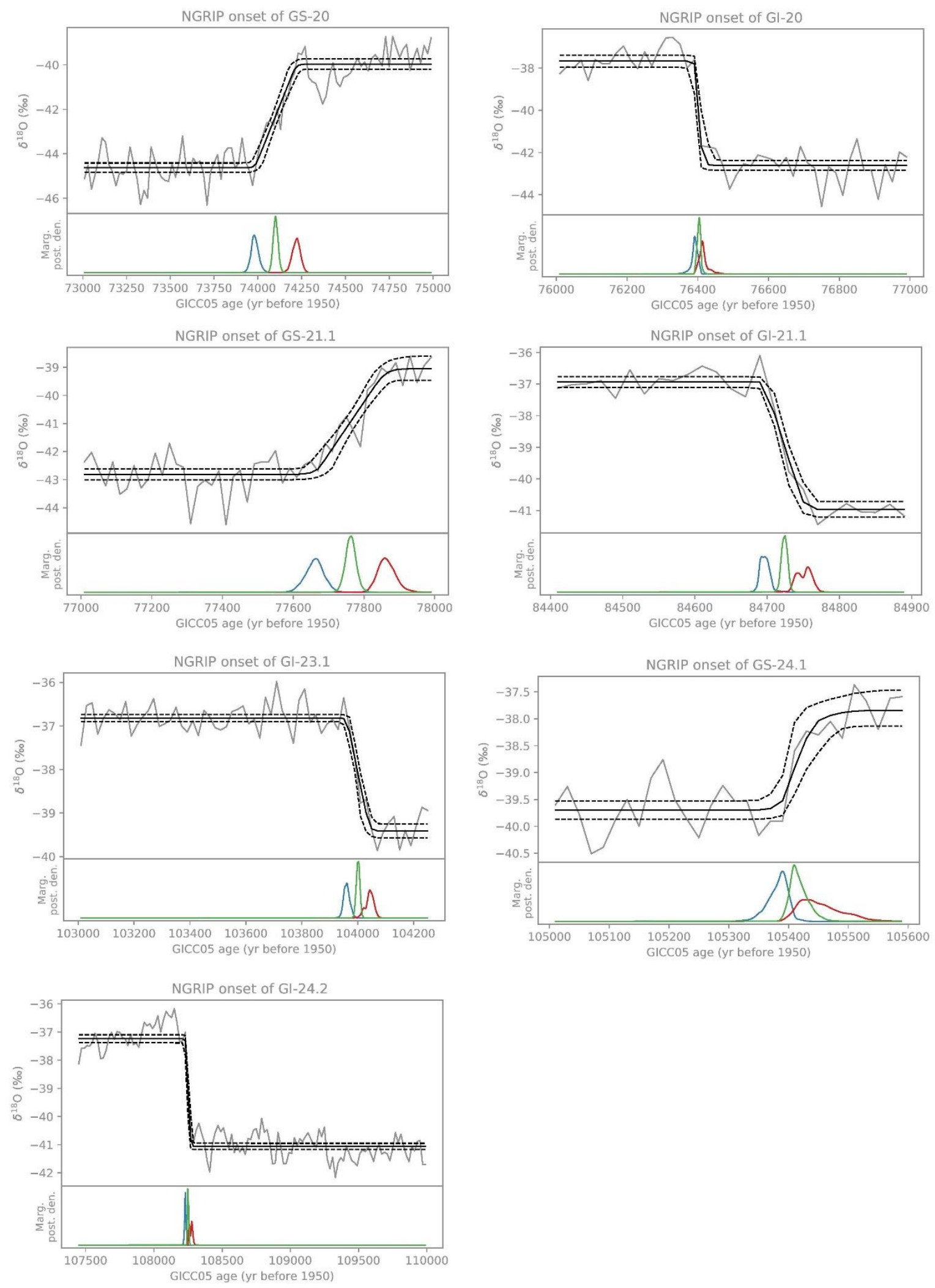

Figure S7: Fitting results of the Erhardt et al. (2019) algorithm for NGRIP on the GICC05modelext chronology (Wolff et al, 2010). Each of the top panels shows the fitted data together with marginal posterior median and 5th and 95th percentile of the fitted ramp in solid and dashed lines respectively. The bottom panels show the marginal posterior densities for the onset, midpoint and endpoint of the transitions (in red, green and blue). 

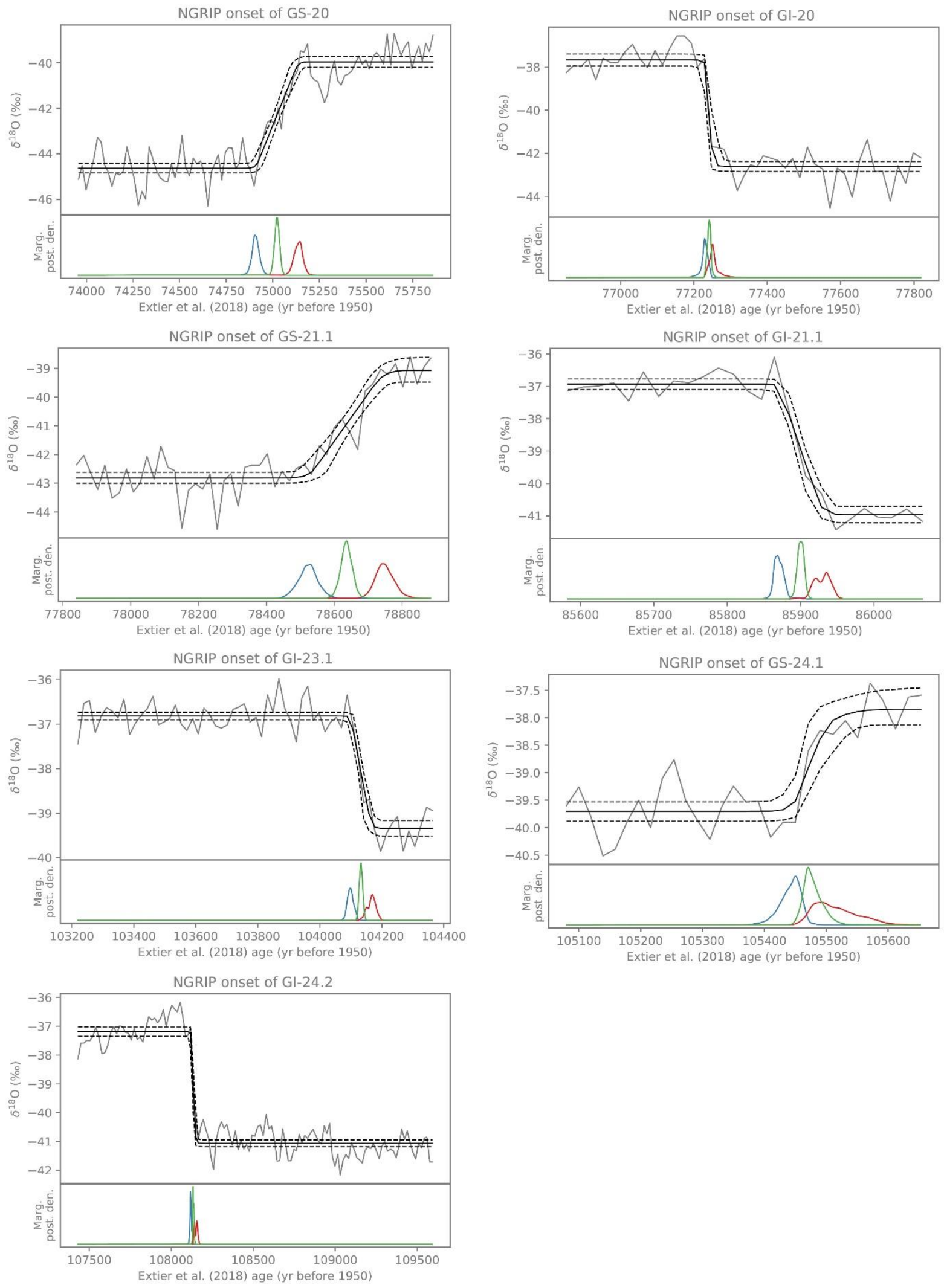

Figure S7: Fitting results of the Erhardt et al. (2019) algorithm for NGRIP on the Extier et al. (2018) revised AICC2012 chronology. Each of the top panels shows the fitted data together with marginal posterior median and 5th and 95th percentile of the fitted ramp in solid and dashed lines respectively. The bottom panels show the marginal posterior densities for the onset, midpoint and endpoint of the transitions (in red, green and blue). 


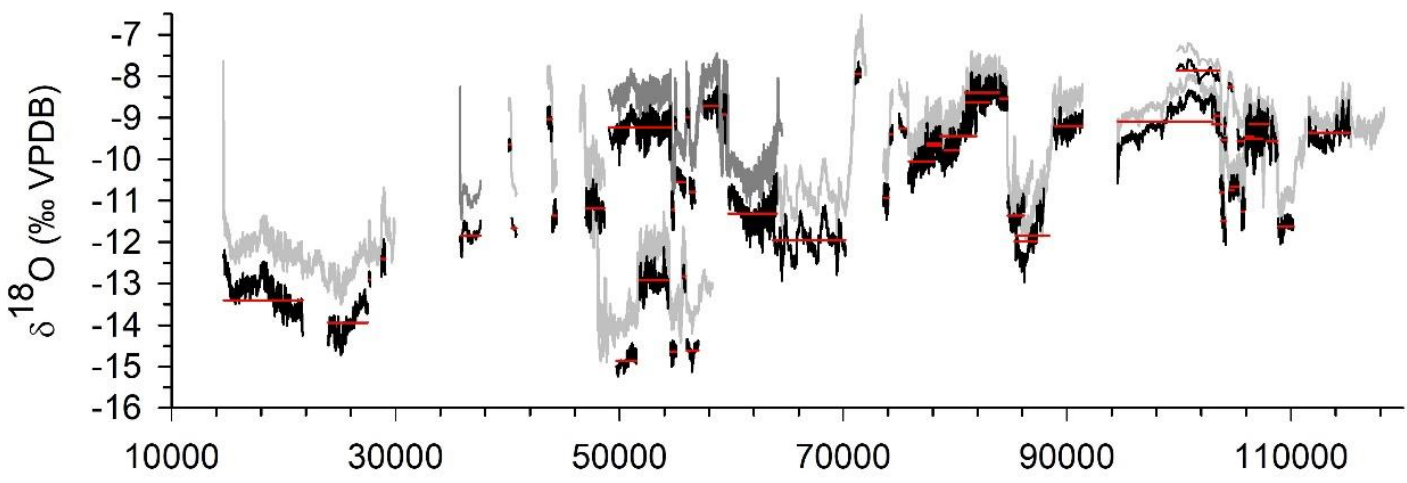

Age (years before 1950 A.D.)

Figure S8: Speleothem $\delta^{18} \mathrm{O}$ records from the northern rim and central European Alps uncorrected for changes in mean $\delta^{18} \mathrm{O}$ variability of seawater (grey). Light grey are single speleothems, dark grey are composite speleothem records. See Fig. 5 for details of the original data. Sections of the ice-volume-corrected $\delta^{18} O$ curve used to establish mean and median $\delta^{18} \mathrm{O}$ values (black) presented in Table S3. Mean values (red horizontal bars).

Table S3: Mean and median $\delta^{18} \mathrm{O}$ values for specific stadials and interstadials as shown in red horizontal bars (Fig. S8). *see Fig. $\$ 9$

\begin{tabular}{|c|c|c|c|c|}
\hline $\begin{array}{c}\text { Equivalent } \\
\text { Stratigraphic } \\
\text { Unit }\end{array}$ & Sample & Longitude & Mean $\delta^{18}$ O & Median $\delta^{18}$ O \\
\hline GS-2.1 & $7 H 3$ & 7.81 & -13.4 & -13.5 \\
\hline GS-3 & $7 H 2$ & 7.81 & -13.9 & -14.0 \\
\hline GI-3 & $7 H 2$ & 7.81 & -12.9 & -12.8 \\
\hline GI-4 & $7 H 2$ & 7.81 & -12.4 & -12.4 \\
\hline GS-8 & HÖL-16/17 & 10.15 & -11.8 & -11.8 \\
\hline Gl-9 & HÖL-7 & 10.15 & -9.65 & -9.64 \\
\hline GS-10 & HÖL-7 & 10.15 & -11.66 & -11.66 \\
\hline GI-11 & HÖL-7 & 10.15 & -9.03 & -9.01 \\
\hline GS-12 & HÖL-7 & 10.15 & -11.36 & -11.33 \\
\hline GS-13 & HÖL-7 & 10.15 & -11.2 & -11.2 \\
\hline GS-14 & SPA-49 & 11.67 & -14.9 & -14.9 \\
\hline GI-14 & HÖL-16/17/18 & 10.15 & -9.2 & -9.2 \\
\hline GI-14 & SPA-49 & 11.67 & -12.9 & -12.9 \\
\hline GS-15.1 & HÖL-16/17/18 & 10.15 & -11.2 & -11.2 \\
\hline GS-15.1 & SPA-49 & 11.67 & -14.6 & -14.6 \\
\hline GI-15.1 & HÖL-16/17/18 & 10.15 & -9.1 & -9.2 \\
\hline GS-15.2 & HÖL-16/17/18 & 10.15 & -10.5 & -10.5 \\
\hline GI-15.2 & HÖL-16/17/18 & 10.15 & -9.0 & -9.0 \\
\hline GI-15.2 & SPA-49 & 11.67 & -12.8 & -12.7 \\
\hline GS-16.1 & HÖL-16/17/18 & 10.15 & -10.8 & -10.8 \\
\hline GS-16.1 & SPA-49 & 11.67 & -14.6 & -14.6 \\
\hline GI-16.1 & HÖL-16/17/18 & 10.15 & -8.7 & -8.7 \\
\hline GI-17.1 & HÖL-16/17/18 & 10.15 & -8.9 & -8.9 \\
\hline GS-18 & HÖL-16/17/18 & 10.15 & -11.3 & -11.3 \\
\hline GS-19.2 & KC-1 & 10.11 & -12.0 & -12.0 \\
\hline & & & & \\
\hline
\end{tabular}




\begin{tabular}{|c|c|c|c|c|}
\hline GI-19.2 & KC-1 & 10.11 & $\begin{array}{l}-7.9 \\
\end{array}$ & -8.0 \\
\hline $\begin{array}{c}\text { Equivalent } \\
\text { Stratigraphic } \\
\text { Unit }\end{array}$ & Sample & Longitude & Mean $\delta^{18} \mathrm{O}$ & Median $\delta^{18} O$ \\
\hline GS-20 & HÖL-19 & 10.15 & -10.9 & -10.9 \\
\hline GI-20a & HÖL-19 & 10.15 & -9.4 & -9.4 \\
\hline $\mathrm{GI}-20 \mathrm{c}$ & $\mathrm{SCH}-6$ & 10.07 & -9.3 & -9.3 \\
\hline GS-21.1 & $\mathrm{SCH}-6$ & 10.07 & -10.1 & -10.0 \\
\hline GI-21.1a & GAS-12 & 13.84 & -9.5 & -9.6 \\
\hline GI-21.1a & GAS-13 & 13.84 & -9.8 & -9.7 \\
\hline GI-21.1c & GAS-12 & 13.84 & -9.4 & -9.6 \\
\hline GI-21.1c & GAS-13 & 13.84 & -9.8 & -9.8 \\
\hline GI-21.1e & BA-7 & 9.67 & -8.4 & -8.4 \\
\hline GI-21.1e & BA-1 & 9.67 & -8.6 & -8.6 \\
\hline GI-21.1e & GAS-25 & 13.84 & -8.5 & -8.6 \\
\hline GS-22 & BA-7 & 9.67 & -11.4 & -11.4 \\
\hline GS-22 & BA-5 & 9.67 & -11.8 & -11.8 \\
\hline GS-22 & BA-1 & 9.67 & -12.0 & -11.9 \\
\hline GI-22 & GAS-25 & 13.84 & -9.2 & -9.2 \\
\hline GI-23.1 & EXC-3 & 7.78 & -8.6 & -8.6 \\
\hline GI-23.1 & $7 \mathrm{H} 12 *$ & 7.81 & -7.9 & -7.8 \\
\hline GI-23.1 & HUN-14 & 12.03 & -9.2 & -9.2 \\
\hline GI-23.1 & GAS-27 & 13.84 & -9.0 & -9.0 \\
\hline GS-23.2 & $7 \mathrm{H} 12^{*}$ & 7.81 & -9.5 & -9.5 \\
\hline GS-23.2 & HUN-14 & 12.03 & -11.5 & -11.5 \\
\hline GS-23.2 & GAS-27 & 13.84 & -10.8 & -10.7 \\
\hline GI-23.2 & $7 \mathrm{H} 12^{*}$ & 7.81 & -8.3 & -8.3 \\
\hline GI-23.2 & GAS-27 & 13.84 & -9.2 & -9.2 \\
\hline GS-24.1 & $7 \mathrm{H} 12$ & 7.81 & -9.6 & -9.6 \\
\hline GS-24.1 & HUN-14 & 12.03 & -11.3 & -11.2 \\
\hline GS-24.1 & GAS-27 & 13.84 & -10.7 & -10.8 \\
\hline GS-24.1 & GAS-29 & 13.84 & -10.7 & -10.7 \\
\hline GI-24.1a & GAS-29 & 13.84 & -9.5 & -9.4 \\
\hline GI-24.1 & EXC-3 & 7.78 & -9.0 & -8.9 \\
\hline GI-24.1 & HUN-14 & 12.03 & -9.5 & -9.5 \\
\hline GI-24.1 & GAS-22 & 13.84 & -9.1 & -9.1 \\
\hline GI-24.2 & EXC-3 & 7.78 & -8.8 & -8.8 \\
\hline GI-24.2 & HUN-14 & 12.03 & -9.6 & -9.6 \\
\hline GS-25 & EXC-4 & 7.78 & -12.5 & -12.5 \\
\hline GS-25 & HUN-14 & 12.03 & -11.6 & -11.6 \\
\hline GI-25 & $\mathrm{SCH}-7$ & 10.07 & -9.4 & -9.4 \\
\hline
\end{tabular}




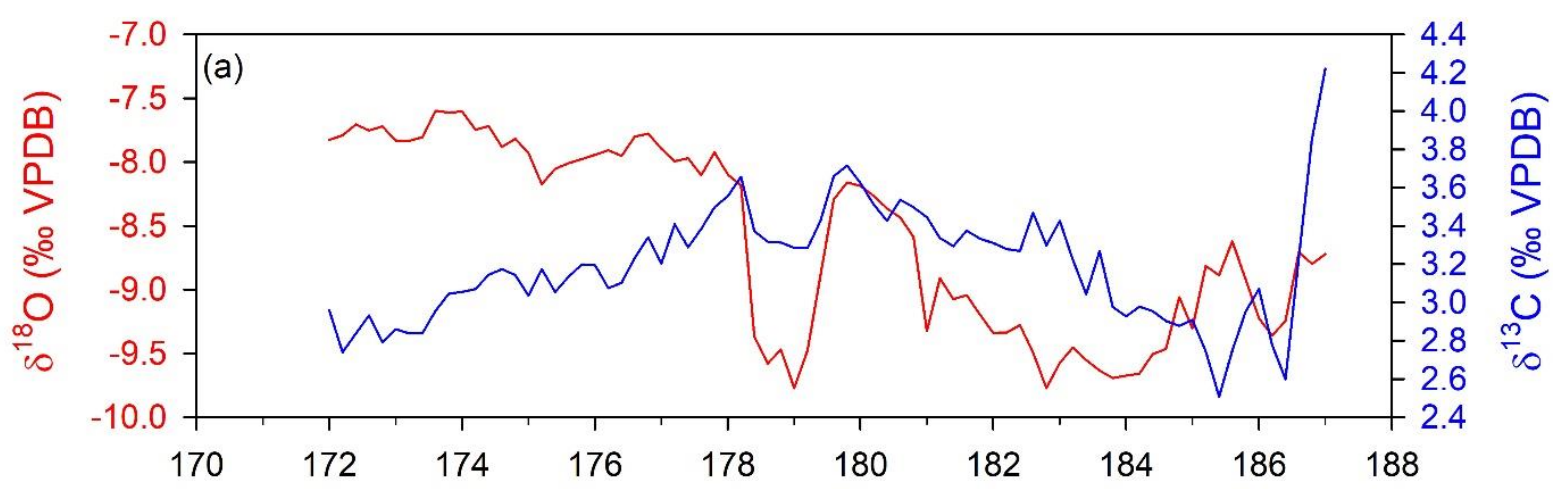

Distance along growth axis ( $\mathrm{mm}$ from top)

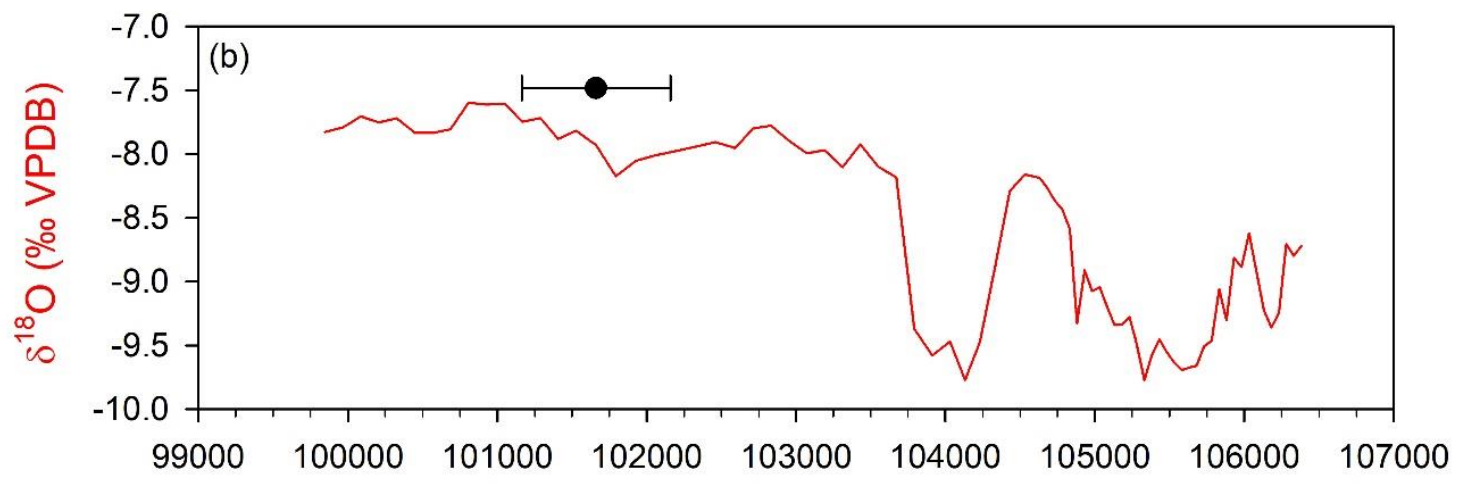

Age (yrs before 1950 A.D.)

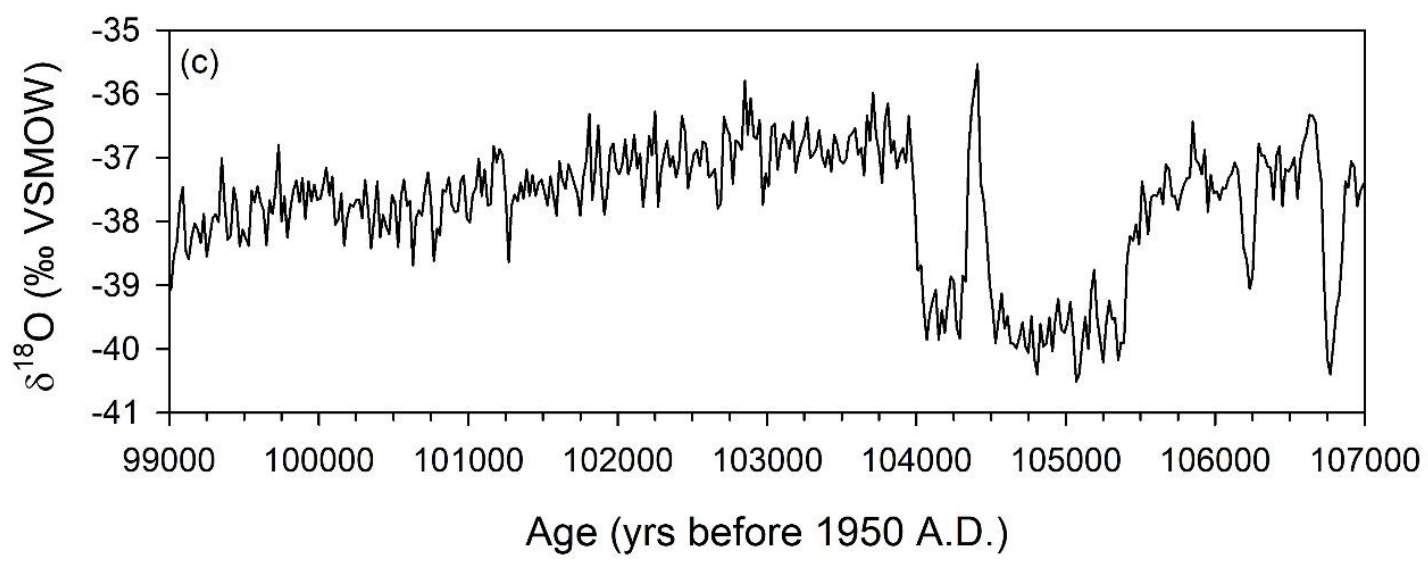

Figure S9: Preliminary data from Siebenhengste sample 7H12 provided by Marc Luetscher for this study. (a) Stable isotope data relative to distance along growth axis. (b) $\delta^{18} \mathrm{O}$ on a preliminary age model. The U-Th age (black circle and 2 sigma uncertainty) firmly places the timing in GI-23.1. Since there are no hiatuses, the remainder is matched to a preliminary age model. The quality of the age model is not so important here because we are only interested in the mean $\delta^{18} \mathrm{O}$ values for the respective stadials and interstadials. (c) NGRIP on GICC05modelext (Wolff et al., 2010). 


\section{References}

Boch, R., Cheng, H., Spötl, C., Edwards, R. L., Wang, X., and Häuselmann, Ph.: NALPS: a precisely dated European climate record 120-60 ka, Clim. Past, 7, 1247-1259, https://doi.org/10.5194/cp-7-1247-2011, 2011.

Cheng, H., Edwards, R. L., Sinha, A., Spötl, C., Yi, L., Chen, S., Kelly, M., Kathayat, G., Wang, X., Li, X., Kong, X., Wang, Y., Ning, Y., and Zhang, H.: The Asian monsoon over the past 640,000 years and ice age terminations, Nature, 534, 640646, https://doi.org/10.1038/nature18591, 2016.

Cheng, H., Edwards, R. L., Shen, C-C., Polyak, V. J., Asmerom, Y., Woodhead, J., Hellstrom, J., Wang, Y., Kong, X., Spötl, C., Wang, X., and Alexander Jr, E. C.: Improvements in ${ }^{230}$ Th dating, ${ }^{230} \mathrm{Th}$ and ${ }^{234} \mathrm{U}$ half-life values, and U-Th isotopic measurements by multi-collector inductively coupled plasma mass spectrometry, Earth Planet. Sc. Lett., 371-372, 82-91, http://dx.doi.org/10.1016/j.eps1.2013.04.006, 2013.

Erhardt, T., Capron, E., Rasmussen, S. O., Schüpbach, S., Bigler, M., Adolphi, F., and Fischer, H.: Decadal-scale progression of the onset of Dansgaard-Oeschger warming events, Clim. Past, 15, 811-825, https://doi.org/10.5194/cp-15-811-2019, 2019.

Extier, T., Landais, A., Bréant, C., Prié, F., Bazin, L., Dreyfus, G., Roche, D. M., and Leuenberger, M.: On the use of $\mathrm{d}^{18}$ Oatm for ice core dating, Quaternary Sci. Rev., 185, 244-257, https://doi.org/10.1016/j.quascirev.2018.02.008, 2018

Jaffey, A. H., Flynn, K. F., Glendenin, L. E., Bentley, W. C., and Essling, A. M.: Precision measurement of half-lives and specific activities of ${ }^{235} \mathrm{U}$ and ${ }^{238} \mathrm{U}$, Phys. Rev. C, 4, 1889, https://doi.org/10.1103/PhysRevC.4.1889, 1979.

Jiang, X., Wang, X., He, Y., Hu, H-M., Li, Z., Spötl, C., and Shen, C-C.: Precisely dated multidecadally resolved Asian summer monsoon dynamics 113.5-86.6 thousand years ago, Quaternary Sci. Rev., 143, 1-12, https://doi.org/10.1016/j.quascirev.2016.05.003, 2016.

Kelly, M. J.: Characterization of Asian Monsoon variability since the Penultimate Interglacial on orbital and sub-orbital timescales, Dongge Cave, China, PhD Thesis, University of Minnesota, USA, 221 pp., 2010.

Kelly, M. J., Edwards, R. L., Cheng, H., Yuan, D., Cai, Y., Zhang, M., Lin, Y., and An, Z.: High resolution characterization of the Asian Monsoon between 146,000 and 99,000 years B.P. from Dongge Cave, China and global correlation of events surrounding Termination II, Palaeogeogr. Palaeocl., 236, 20-38, https://doi.org/10.1016/j.palaeo.2005.11.042, 2006.

Moseley, G. E., Spötl, C., Svensson, A., Cheng, H., Brandstätter, S., and Lawrence Edwards, R. L.: Multi-speleothem record reveals tightly coupled climate between central Europe and Greenland during Marine Isotope Stage 3, Geology, 42, 10431046, https://doi.org/10.1130/G36063.1, 2014.

Svensson, A., Andersen, K. K., Bigler, M., Clausen, H. B., Dahl-Jensen, D., Davies, S. M., Johnsen, S. J., Muscheler, R., Parrenin, F., Rasmussen, S. O., Röthlisberger, R., Seierstad, I., Steffensen, J. P., and Vinther, B. M.: A 60000 year Greenland stratigraphic ice core chronology, Clim. Past, 4, 47-57, https://doi.org/10.5194/cp-4-47-2008, 2008.

Veres, D., Bazin, L., Landais, A., Toyé Mahamadou Kele, H., Lemieux-Dudon, B., Parrenin, F., Martinerie, P., Blayo, E., Blunier, T., Capron, E., Chappellaz, J., Rasmussen, S. O., Severi, M., Svensson, A., Vinther, B., and Wolff, E. W.: The Antarctic ice core chronology (AICC2012): an optimized multi-parameter and multi-site dating approach for the last 120 thousand years, Clim. Past, 9, 1733-1748, https://doi.org/10.5194/cp-9-1733-2013, 2013.

Wang, Y. J. Cheng, H., Edwards, R.L., Kong, X., Shao, X., Cheng, S., Wu, J., Jiang, X., Wang, X., and An, Z.: Millennial- and orbital- scale changes in the East Asian Monsoon over the past 224,000 years, Nature, 451, 1090-1093, https://doi.org/10.1038/nature06692, 2008.

Wedepohl, K. H.: The composition of the continental crust, Geochim. Cosmochim. Ac., 59, 1217-1239, https://doi.org/10.1016/0016-7037(95)00038-2, 1995.

Wolff, E. W., Chappellaz, J., Blunier, T., Rasmussen, S. O., and Svensson, A.: Millennial-scale variability during the last glacial: The ice core record, Quaternary Sci. Rev., 29, 2828-2838, https://doi.org/10.1016/j.quascirev.2009.10.013, 2010. 